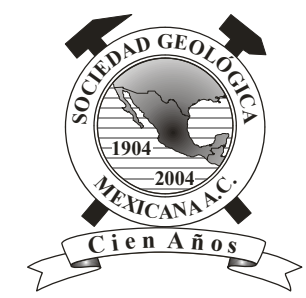

\title{
Mineralogía magnética de suelos volcánicos en una toposecuencia del valle de Teotihuacán
}

\author{
Jorge F. Rivas Ortiz ${ }^{1, *}$, Beatriz Ortega Guerrero², Elizabeth Solleiro Rebolledo ${ }^{3}$, \\ Sergey Sedov ${ }^{3}$, Serafín Sánchez Pérez ${ }^{4}$ \\ ${ }^{1}$ Instituto de Geofísica, Posgrado en Ciencias de la Tierra, Universidad Nacional Autónoma de México, Ciudad Universitaria, 04510, \\ México D.F., México \\ ${ }^{2}$ Instituto de Geofísica, Departamento de Geomagnetismo y Exploración, Universidad Nacional Autónoma de México, Ciudad \\ Universitaria, 04510, México D.F., México \\ ${ }^{3}$ Instituto de Geología, Departamento de Edafología, Universidad Nacional Autónoma de México, Ciudad Universitaria, 04510, \\ México D.F., México \\ ${ }^{4}$ Instituto de Geología, Posgrado en Ciencias de la Tierra, Ciudad Universitaria, Universidad Nacional Autónoma de México, 04510, \\ México D.F., México \\ *jorger@geofisica.unam.mx
}

\begin{abstract}
Resumen
Numerosos trabajos han demostrado la relación existente entre la mineralogía magnética de suelos y paleosuelos y las variaciones climáticas y ambientales, o el efecto de la contaminación en los mismos. Con el propósito de analizar esta relación en una toposecuencia del valle de Teotihuacán, se llevó a cabo un estudio de las propiedades magnéticas en cinco suelos y un paleosuelo desarrollados en depósitos volcanoclásticos distribuidos en diferentes posiciones del relieve entre 2250 y 3060 msnm, que incluyen: dos Cambisoles, un Calcisol, dos Fluvisoles y un Vertisol. La mineralogía magnética fue caracterizada a través de técnicas de magnetismo de rocas que determinan la composición, concentración y distribución de tamaños de dominio de los minerales magnéticos, y esta caracterización fue comparada y validada con parámetros no magnéticos. Los resultados de la investigación muestran que existe una marcada diferenciación de las propiedades magnéticas en función de las condiciones ambientales de cada suelo y su grado de pedogénesis. Las características magnéticas varían entre dos extremos: 1) los Cambisoles contienen mayor concentración de minerales magnéticos de mayor tamaño; y 2) los Fluvisoles presentan bajas concentraciones de minerales magnéticos dominados por partículas finas. El Calcisol presenta variaciones entre estos dos extremos, tanto en la concentración de minerales magnéticos como en su tamaño, mientras que el Vertisol presenta baja concentración y tamaños gruesos. A diferencia de lo que sucede en las secuencias de loess-paleosuelos que registran un aumento en susceptibilidad magnética $(\chi)$ en la parte superficial, originado por un incremento de minerales ferrimagnéticos y antiferrimagnéticos de tamaño fino DS y SP, los realces observados en un Cambisol y un Fluvisol de Teotihuacán se deben a la incorporación de nuevos materiales de origen volcánico y fluvial en la superficie. Por el contrario, las mayores concentraciones de partículas SP y DS ocurren en general en los horizontes inferiores. En ellos es débil aún la formación de minerales magnéticos pedogénicos, tanto ferrimagnéticos como antiferrimagnéticos. La baja magnetización intrínseca de estos últimos y la proporción relativamente baja en la que se presentan ocasionan que sea difícil su detección, ya que quedan enmascarados por las fases ferrimagnéticas. Nuestros análisis muestran las particularidades de suelos desarrollados en materiales volcánicos y el potencial de los estudios de propiedades magnéticas en combinación con otros indicadores pedogenéticos en las reconstrucciones de las condiciones ambientales en el pasado.
\end{abstract}

Palabras clave: mineralogía magnética, suelos volcánicos, toposecuencia, valle de Teotihuacán. 


\begin{abstract}
Numerous studies have demonstrated the relationship between magnetic mineralogy in soil and paleosol sequences and climatic and environmental variations as well as pollution effects affecting them. In order to analyze this relationship, we carried out a study of magnetic properties in five soils and a paleosol, all developed on volcaniclastic deposits and located throughout an altitudinal range of 2250 to $3040 \mathrm{~m}$ above sea level and within a toposequence from Teotihuacan valley. These soil profiles consist of two Cambisols, a Calcisol, two Fluvisols, as well as a Vertisol. The magnetic mineralogy was characterized by rock magnetism techniques in order to determine the composition, concentration and domain size distribution of magnetic minerals, and was compared and validated with other non-magnetic parameters. Results reflect a clear difference in magnetic properties which are influenced by environmental conditions for each soil and their degree of pedogenesis. These magnetic properties vary between two extremes: 1) Cambisols, with higher concentrations of magnetic minerals of larger particle size; and 2) Fluvisols with low concentrations of magnetic minerals dominated by fine particle size. The analyzed Calcisol presents variations between these two extremes, for both aspects (concentration and grain sizes), while the Vertisol shows low concentration and coarse sizes. Unlike magnetic susceptibility enhancement reported in loess-paleosol sequences, which is caused by a rise of SD and SP ferri-and antiferrimagnetic minerals, the increase reflected in a Cambisol and Fluvisol from Teotihuacan is caused by the incorporation of new volcanic and fluvial material to the upper part of the soils. By contrast, the higher SP and SD concentrations are located in the lower horizons. These present a rather weak formation of pedogenic magnetic minerals, both ferrimagnetic and antiferrimagnetic. The low intrinsic magnetization of the latter and the relatively low proportion in which they occur make identification difficult because they are masked by ferrimagnetic phases. Our analysis highlights the features of soils developed on volcanic material as well as the potential that the analysis of magnetic properties holds in combination with other pedogenetic indicators for the reconstruction of past environmental conditions.
\end{abstract}

Keywords: magnetic mineralogy, volcanic soils, toposequence, Teotihuacan valley.

\section{Introducción}

Numerosos trabajos han tratado de establecer las relaciones entre las variaciones climáticas ocurridas durante el Cuaternario y la mineralogía magnética de suelos y paleosuelos de diversas localidades en el mundo. Estos estudios han sido particularmente prometedores en las secuencias de loess-paleosuelos de China (Liu et al., 2007, Lu et al., 2008), Europa (Oaches y Banerjee, 1996; Jordanova et al., 2003, 2004; Liu et al., 2010), Argentina (Orgeira et al., 2003; Bidegain et al., 2009) y el noroeste de Estados Unidos (Geiss et al., 2008). En otros trabajos se ha establecido la relación entre la mineralogía magnética de suelos y cambios ambientales (Grimley et al., 2004; Geiss y Zanner, 2006, 2007) y contaminación (Chaparro, 2006; Orgeira et al., 2008), entre otros. Muchos estudios de este tipo se basan únicamente en la susceptibilidad magnética $(\chi)$ como indicador ( o proxy ) de las variaciones climáticas y ambientales. Sin embargo, ya que este parámetro depende no sólo de la concentración de partículas ferrimagnéticas, sino también de la presencia de un amplio rango de minerales magnéticos y de sus distribuciones de tamaño (Geiss y Zanner, 2006), la interpretación de la $\chi$ rara vez es única y directa, por lo que las interpretaciones pueden verse comprometidas si no se han caracterizado adecuadamente los componentes magnéticos. Por ello, los análisis de magnetismo de rocas deben involucrar técnicas que determinen la composición, concentración y distribución de tamaños de dominio de los minerales magnéticos, y frecuentemente requieren de información adicional de otros parámetros no magnéticos para lograr una acertada interpretación paleoambiental.

Gran parte de las secuencias de loess-paleosuelos se caracterizan por registrar un aumento en $\chi$ en la parte superficial del suelo. Este aumento se debe mayormente a un incremento de minerales ferrimagnéticos ( magnetita y/o maghemita ) de tamaño ultrafino superparamagnético (SP, $<0.01 \mu \mathrm{m}$ ) a dominio sencillo (DS, $<0.1 \mu \mathrm{m}$; p.ej. Maher, 1986; Heller y Evans, 1995; Hunt et al., 1995), pero también al incremento de partículas tanto ferrimagnéticas como antiferrimagnéticas ( hematita o goethita ) (Geiss et al., 2004). El origen de dicho incremento se ha interpretado de diferentes maneras, entre ellas la ocurrencia de incendios, fermentación, calentamiento, actividad biológica (Tite y Linington, 1975; Mullins, 1977; Maher, 1986; Thompson y Oldfield, 1986), la contaminación del suelo (Chaparro, 2006) y la transformación de minerales paramagnéticos o antiferrimagnéticos a fases ferrimagnéticas (Geiss y Zanner, 2007).

Este incremento en $\chi$ ha sido utilizado para reconstruir las condiciones paleoclimáticas durante la formación de los suelos (Verosub et al., 1993, entre otros). A partir de las primeras observaciones hechas para definir la influencia del clima, particularmente la precipitación, en la concentración y el tipo de minerales magnéticos (Maher et al., 1994; Han et al., 1996; Maher, 1998), se generó una controversia sobre el efecto real de la precipitación en la formación de los minerales magnéticos pedogenéticos. Porter et al. (2001) señalan que el efecto de la lluvia en la señal magnética sólo es responsable del 10 al $11 \%$ de la 
varianza de la susceptibilidad magnética en suelos loésicos. Sin embargo, Maher et al. (2002), en un estudio detallado en las estepas rusas, establecen que la precipitación tiene un papel fundamental en la distribución de los minerales magnéticos del suelo. Por otra parte, Bartel et al. (2011) encuentran en suelos loésicos de la región sur de las pampas argentinas una diferenciación en las propiedades magnéticas de acuerdo a su grado de evolución y gradiente de humedad.

En numerosos suelos y paleosuelos volcánicos o loésicos con contribución de minerales volcánicos el comportamiento que se ha observado es opuesto a lo encontrado en las secuencias de loess de China y Rusia, ya que no presentan el aumento en $\chi$ en la parte superficial (Orgeira et al., 1998; Vásquez et al., 1998; Rivas-Ortiz, 2003; Schellenberger et al., 2003; Ortega-Guerrero et al., 2004; Rivas-Ortiz, 2006; Rivas-Ortiz et al., 2006; entre otros). Sin embargo, en ellos también se reconoce que la pedogénesis es responsable de tal comportamiento. Ortega-Guerrero y colaboradores (2004) establecen que la ausencia del realce magnético se debe a un balance negativo entre los procesos pedogénicos que son los responsables de la formación de una mineralogía magnética ultrafina y las condiciones reductomórficas bajo climas húmedos que resultan en la destrucción de los mismos. De hecho, el hidromorfismo en los suelos causa una disminución en $\chi$ (Nawrocki et al., 1996; Terhorst et al., 2001; Grimley y Vepraskas, 2000; Grimley et al., 2004). Por otra parte, la composición del material parental tiene un papel fundamental en la ausencia del aumento de susceptibilidad magnética, ya que la abundancia de minerales ferrimagnéticos en depósitos volcaniclásticos básicos e intermedios es tal que la pedogénesis no compensa de manera eficiente la pérdida de los minerales primarios.

Con el propósito de establecer la relación entre la mineralogía magnética y el clima, se analizan las propiedades magnéticas en una toposecuencia compuesta por cinco perfiles de suelos volcánicos modernos y un paleosuelo en el valle de Teotihuacán. Este estudio contribuye al desarrollo de una función de transferencia que pueda ser aplicada a los paleosuelos volcánicos con el objetivo de establecer un modelo de variabilidad climática en el centro de México y las condiciones ambientales bajo las que se desarrollan los suelos de esta región.

\section{2. Área de estudio}

El valle de Teotihuacán se localiza en la porción centrooriental del Cinturón Volcánico Transmexicano dentro de la cuenca de México (Figura 1). Su altitud oscila entre 2250 y $3060 \mathrm{msnm}$. Geomorfológicamente, el valle se caracteriza por presentar una extensa altiplanicie escalonada así como por la presencia de volcanes aislados (Lugo Hubp, 1984). Las rocas volcánicas más antiguas en la región son andesitas y dacitas del Plioceno temprano y basaltos del Plioceno tardío, que afloran en el cerro Patlachique (Vázquez-Sánchez y Jaimes-Palomera, 1989) y el cerro

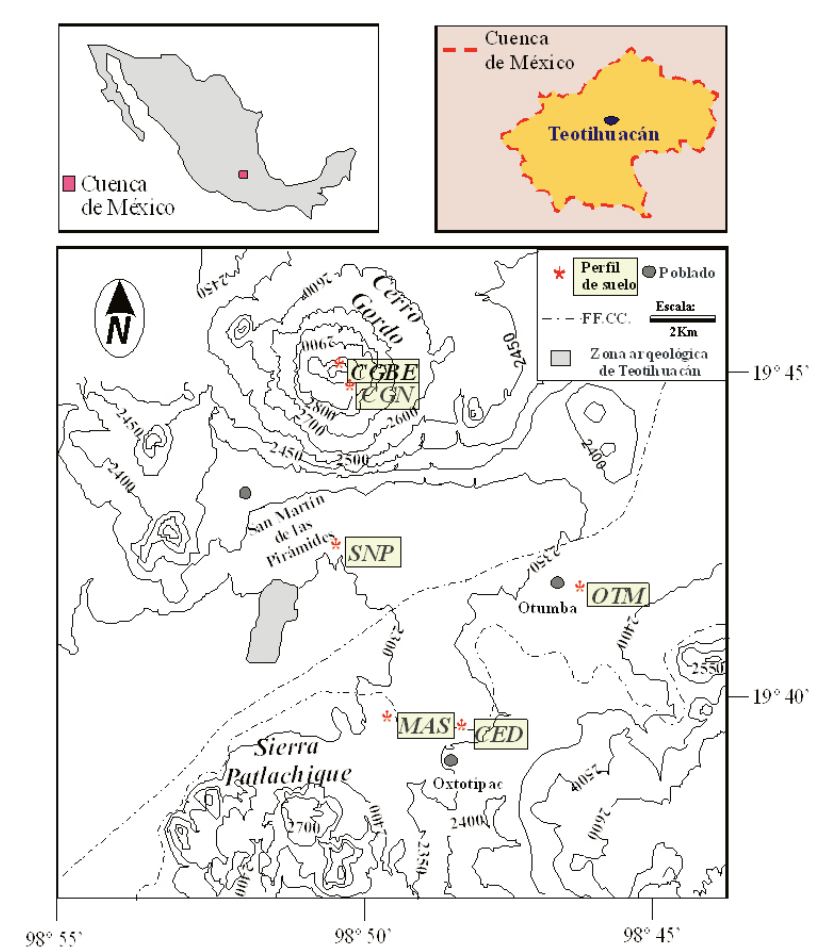

Figura 1. Localización de los seis suelos volcánicos que se estudiaron dentro de la región del valle de Teotihuacán. Los perfiles CGBE y CGN son Cambisoles, MAS es un Calcisol, los perfiles SNP y OTM son Fluvisoles y el perfil CED es un Vertisol.

Gordo (García-Palomo et al., 2002).

Los suelos del valle son de tipo Fluvisol, Feozem, Cambisol, Leptosol y Vertisol (INEGI, 1999). La orografía afecta a la precipitación, lo que da lugar a climas que varían de semisecos a subhúmedos, registrándose una precipitación anual de $563.3 \mathrm{~mm}$ y una temperatura media anual de 14.9 ${ }^{\circ} \mathrm{C}$ (García, 1968, 1988). De acuerdo a las estimaciones de Castilla y Tejero (1983), el clima de Teotihuacán varía en función de la altitud, de manera que en las posiciones más elevadas, en el cerro Gordo, la precipitación anual es de $800 \mathrm{~mm}$ con una temperatura media anual de $12{ }^{\circ} \mathrm{C}$, como consecuencia de la sombra parcial y la dirección de los vientos, sobre todo en la ladera norte (McClung de Tapia et al., 2003).

Los suelos analizados en esta investigación han sido estudiados previamente por otros autores en estudios paleoambientales mediante diversas técnicas (CabadasBáez, 2004, 2007; Gama-Castro et al., 2005a, 2005b; Lounejeva Baturina et al., 2007a, 2007b; Solleiro-Rebolledo et al., 2006, 2011), y en estudios geoarqueológicos (McClung de Tapia et al., 2003, 2005; Rivera-Uria et al., 2007; RiveraUria, 2008). Se seleccionaron seis perfiles distribuidos a lo largo de una toposecuencia de aproximadamente $10 \mathrm{~km}$, con dirección NW-SE (Figura 2, Tabla 1). La clasificación de los suelos fue establecida con base en los criterios de la WRB (2006) y tomada de los trabajos previos (McClung de Tapia et al., 2003; Solleiro-Rebolledo et al., 2006; Rivera- 


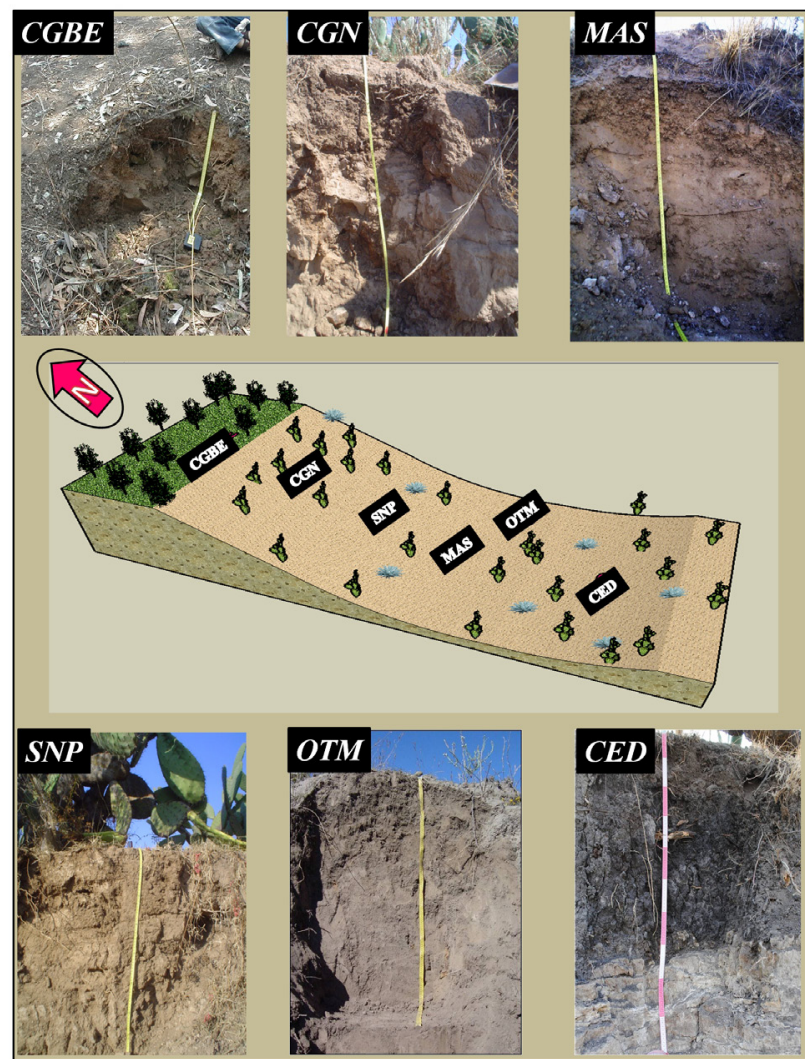

Figura 2. Representación esquemática de la ubicación de los perfiles de suelo estudiados dentro de la toposecuencia.
Uria et al., 2007). Los perfiles de suelos estudiados son: cerro Gordo (CGBE y CGN), ubicados en las posiciones de mayor altitud (Tabla 1); Maseca (MAS), en el talud inferior, en la transición entre el cerro y el valle; San Pablo (SNP) y Otumba (OTM), en las posiciones más bajas, representando los perfiles del fondo del valle. Adicionalmente, se analizó un perfil denominado Cedazo (CED), el cual contiene un paleosuelo de color oscuro, que se correlaciona con un paleosuelo sepultado en San Pablo. En esta localidad, este último se encuentra a una profundidad de $1.25 \mathrm{~m}$, separado de SNP por sedimentos y pedosedimentos aluviales (RiveraUria et al., 2007; Solleiro-Rebolledo et al., 2011).

\subsection{Descripción de los perfiles}

A continuación se presenta, de acuerdo a su posición en la toposecuencia, una breve descripción de los perfiles analizados. Las edades de los suelos se estiman de acuerdo con los fechamientos de ${ }^{14} \mathrm{C}$ publicados en trabajos previos y se presentan como edades calibradas antes del presente (cal AP). En todos los perfiles el material parental consiste en depósitos volcanosedimentarios de composición andesítica-basáltica. Cabadas-Báez (2004) reporta en CGN, MAS y OTM una asociación predominante de plagioclasas intermedias (50-62\%), ferromagnesianos (20-30\%) y cuarzo (5-10\%), que coinciden con las observaciones de Rivera-Uria (2008) en SNP.

Tabla 1. Localización de los perfiles estudiados dentro del valle y algunas de sus características ambientales.

\begin{tabular}{|c|c|c|c|c|c|c|c|c|c|}
\hline Localidad/Perfil & $\begin{array}{c}\text { Ubicación } \\
\text { Coordenadas } \\
\text { Altitud (m) } \\
\end{array}$ & Horizontes & $\begin{array}{c}\text { Edad } \\
\text { (años cal } A P)\end{array}$ & $\begin{array}{l}\text { Clasificación } \\
\text { (WRB, 2006) }\end{array}$ & Vegetación & Drenaje & Pendiente & $\begin{array}{c}\begin{array}{c}\text { Precipitación } \\
\text { anual }\end{array} \\
(\mathrm{mm})\end{array}$ & $\begin{array}{c}\text { Temperatura } \\
\text { media anual } \\
\\
\left({ }^{\circ} \mathrm{C}\right)\end{array}$ \\
\hline Cerro Gordo CGBE & $\begin{array}{c}19^{\circ} 45^{\prime} 5.4^{\prime \prime} \\
98^{\circ} 49^{\prime} 6.4^{\prime \prime} \\
3040 \mathrm{~m}\end{array}$ & $\mathrm{Ah}_{1}-\mathrm{Ah}_{2}-\mathrm{B}$ & -- & Cambisol & Bosque de encino & Bueno & $8^{\circ}$ & 800 & 12 \\
\hline $\begin{array}{c}\text { Cerro Gordo } \\
\text { CGN }\end{array}$ & $\begin{array}{c}19^{\circ} 44^{\prime} 55^{\prime \prime} \\
98^{\circ} 49^{\prime} 23^{\prime \prime} \\
2948 \mathrm{~m}\end{array}$ & Ap-Ah-AB-BC & $6054 \pm 99^{1}$ & Cambisol & $\begin{array}{c}\text { Cultivo de } \\
\text { Opuntia }\end{array}$ & Moderado & $8.5^{\circ}$ & 750 & 12 \\
\hline $\begin{array}{c}\text { Maseca } \\
\text { MAS }\end{array}$ & $\begin{array}{c}19^{\circ} 39^{\prime} 36.8^{\prime \prime} \\
98^{\circ} 49^{\prime} 19.4^{\prime \prime} \\
2315 \mathrm{~m}\end{array}$ & Ap-Bk-C & $<13358 \pm 140^{2}$ & Calcisol & $\begin{array}{c}\text { Estrato herbáceo } \\
\text { de gramíneas }\end{array}$ & Bueno & $2.4^{\circ}$ & 604 & 16 \\
\hline San Pablo SNP & $\begin{array}{c}19^{\circ} 42^{\prime} 30.9^{\prime \prime} \\
98^{\circ} 9^{\prime} 15.2^{\prime \prime} \\
2317 \mathrm{~m}\end{array}$ & Ap-C & $<520^{3}$ & Fluvisol & $\begin{array}{c}\text { Cultivo de } \\
\text { Opuntia }\end{array}$ & Bueno & $1^{\circ}$ & 608 & 15 \\
\hline Otumba OTM & $\begin{array}{c}19^{\circ} 41^{\prime} 24.5^{\prime \prime} \\
98^{\circ} 45^{\prime} 48.1^{\prime \prime} \\
2371 \mathrm{~m}\end{array}$ & Ap-AB & $<3044 \pm 93^{4}$ & Fluvisol & $\begin{array}{c}\text { Cultivo de } \\
\text { Opuntia }\end{array}$ & Moderado & $2.5^{\circ}$ & 514.3 & 14.8 \\
\hline $\begin{array}{c}\text { Cedazo } \\
\text { CED }\end{array}$ & $\begin{array}{c}19^{\circ} 38^{\prime} 32.6^{\prime \prime} \\
98^{\circ} 48^{\prime} 14.1^{\prime \prime} 2353 \\
\mathrm{~m}\end{array}$ & $\mathrm{Ap}-\mathrm{Ah}_{1}-\mathrm{Ah}_{2}$ & $3166 \pm 69^{5}$ & Vertisol & $\begin{array}{l}\text { Cultivo de } \\
\text { Zea mays }\end{array}$ & Bueno & $7^{\circ}$ & 604 & 16 \\
\hline
\end{tabular}

${ }^{1}$ McClung et al. (2003); ${ }^{2}$ McClung et al. (2005); ${ }^{3}$ Rivera-Uria et al., 2007 


\subsubsection{Cima del cerro Gordo (CGBE)}

Se ubica en la cima del cerro Gordo bajo un bosque de encino y con buen drenaje. Su espesor es de $0.30 \mathrm{~m}$ y presenta una secuencia de horizontes $\mathrm{Ah}_{1}-\mathrm{Ah}_{2}-\mathrm{Bw}$, clasificado como un Cambisol (Figura 2, Tabla 1). El horizonte $\mathrm{Ah}\left(\mathrm{Ah}_{1}\right.$ y $\left.\mathrm{Ah}_{2}\right)$ tiene un color pardo obscuro, así como una densidad de raíces de media a alta, lo que se refleja en su alto contenido de carbono orgánico (5.5\%). Su textura es limosa, aunque en Bw se registra un incremento de arcilla y un cambio de coloración, hacia tonos amarillentos (Tabla 2). El límite entre los subhorizontes es irregular y difuso, con una transición gradual. No se tienen datos de su edad.

\subsubsection{Ladera del cerro Gordo (CGN)}

Se localiza sobre una ladera en el flanco sur del cerro Gordo, en una posición inestable en la cual el bosque de encino ha sido reemplazado por cultivo de Opuntia; presenta drenaje moderado (Figura 2, Tabla 1). El perfil tiene un espesor de $0.71 \mathrm{~m}$, presenta una secuencia de horizontes Ap-Ah-AB-BC y ha sido clasificado como un Cambisol. Anteriormente, Solleiro-Rebolledo et al. (2006) lo clasificaron como un Feozem, aunque en realidad no presenta horizontes de diagnóstico y el horizonte superficial no es mólico. Es un suelo de desarrollo moderado, limoso, aunque con proporciones mayores de arcilla que CGBE (Tabla 2). Es notorio el cambio de color entre el horizonte Ap y el Ah, lo cual se atribuye al uso de suelo, deforestación y cultivación, (Cabadas-Báez, 2004), aunque se observa que el porcentaje de carbono orgánico es igual en ambos horizontes (1.6\%, Tabla 2). Un índice del grado de pedogénesis lo brinda la relación $\mathrm{Feo} / \mathrm{Fed}$ (el cociente entre el hierro extraído con oxalato que evidencia el contenido de hierro amorfo y el hierro extraído con ditionito que indica la presencia de hierro cristalino), de tal forma que entre más pequeño sea este cociente, se refleja una mayor contribución de los minerales de alta cristalinidad. En esta relación, los horizontes $\mathrm{BC}$ y Ap tienen una mayor proporción de óxidos de hierro amorfo, mientras que en los horizontes $\mathrm{Ah}$ y $\mathrm{AB}$ es mayor el contenido de hierro cristalino (Tabla 2). De acuerdo con McClung de Tapia et al. (2003), el paleosuelo que subyace directamente al suelo moderno tiene una edad de 6054 años cal AP, que sugiere la edad máxima del suelo moderno.

\subsubsection{Maseca (MAS)}

Ubicado en la parte baja de la sierra Patlachique en una posición estable del valle y con un buen drenaje. Su espesor es de $1.20 \mathrm{~m}$, donde fueron distinguidos los siguientes horizontes: Ap, Bk, C; ha sido clasificado como un Calcisol (Figura 2, Tabla 1). El horizonte Ap es de color pardo oscuro y contiene abundantes restos de cerámica, pequeños y desgastados, mostrando un uso intensivo desde la época teotihuacana (periodo Clásico, 200-600 D.C.; CabadasBáez, 2004). En el horizonte Bk, el color cambia a pardo amarillento. El suelo tiene una moderada proporción de

Tabla 2.Propiedades selectas de los suelos que conforman la toposecuencia.

\begin{tabular}{|c|c|c|c|c|c|c|c|c|c|c|c|}
\hline Perfil & Horizonte & $\begin{array}{c}\text { Color } \\
\text { Húmedo }^{*}\end{array}$ & $\begin{array}{c}\text { Arena } \\
(\%) \\
\end{array}$ & $\begin{array}{c}\text { Limo } \\
(\%)\end{array}$ & $\begin{array}{c}\text { Arcilla } \\
(\%)\end{array}$ & $p H$ & $\begin{array}{l}\text { C.O. } \\
(\%)\end{array}$ & $\begin{array}{l}\text { Fed } \\
(\%) \\
\end{array}$ & $\begin{array}{l}F e o \\
(\%) \\
\end{array}$ & Feo/Fed & Ref. \\
\hline \multirow{3}{*}{ CGBE } & $\mathrm{Ah}_{1}$ & $7.5 \mathrm{YR} 3 / 3$ & 25.5 & 61.3 & 13.2 & 6 & 5.5 & nd & nd & nd & 1 \\
\hline & $\mathrm{Ah}_{2}$ & $7.5 \mathrm{YR} 3 / 3$ & 27.5 & 55.3 & 17.3 & 6 & 2.4 & nd & nd & nd & 1 \\
\hline & $\mathrm{Bw}$ & $7.5 \mathrm{YR} 3 / 3$ & 27.2 & 49.6 & 23.2 & 6 & 1.7 & nd & nd & nd & 1 \\
\hline \multirow{4}{*}{ CGN } & Ap & $10 \mathrm{YR} 4 / 3$ & 50.08 & 35.56 & 15.36 & 7 & 1.6 & 0.11 & 0.1 & 0.91 & 2 \\
\hline & $\mathrm{Ah}$ & $7.5 \mathrm{YR} 4 / 3$ & 23.92 & 44.67 & 32.03 & 7 & 1.6 & 0.13 & 0.03 & 0.23 & 2 \\
\hline & $\mathrm{AB}$ & $10 \mathrm{YR} 2 / 2$ & 15.57 & 54.75 & 29.68 & 7 & 1 & 0.14 & 0.04 & 0.29 & 2 \\
\hline & $\mathrm{BC}$ & $10 \mathrm{YR} 3 / 3$ & 18.3 & 46.32 & 33.36 & 7 & 0.5 & 0.13 & 0.03 & 0.91 & 2 \\
\hline \multirow{3}{*}{ MAS } & Ap & $10 \mathrm{YR} 3 / 3$ & 37.98 & 36.1 & 25.92 & 8 & 0.8 & 0.3 & 0.2 & 0.67 & 2 \\
\hline & $\mathrm{Bk}$ & $10 \mathrm{YR} 3 / 3$ & 34.47 & 32.79 & 32.74 & 8 & 0.6 & 0.45 & 0.15 & 0.33 & 2 \\
\hline & $\mathrm{C}$ & $10 \mathrm{YR} 3 / 3$ & 24.39 & 48.69 & 26.92 & 8 & 0.4 & 0.35 & 0.13 & 0.37 & 2 \\
\hline \multirow{2}{*}{ SNP } & Ap & $10 \mathrm{YR} 3 / 3$ & 46.8 & 28.2 & 24.9 & 8 & 1 & 0.47 & 0.01 & 0.02 & 3 \\
\hline & $\mathrm{C}$ & $10 \mathrm{YR} 3 / 3$ & 36.2 & 26.9 & 36.9 & 8 & 0.8 & 0.41 & 0.01 & 0.02 & 3 \\
\hline \multirow{2}{*}{ OTM } & Ap & $10 \mathrm{YR} 2 / 2$ & 35.94 & 35.86 & 28.2 & 7 & 1.3 & 0.64 & 0.4 & 0.62 & 2 \\
\hline & $\mathrm{AB}$ & $10 \mathrm{YR} 2 / 2$ & 24.78 & 43.2 & 32.02 & 7 & 1.5 & 0.94 & 0.73 & 0.77 & 2 \\
\hline \multirow{2}{*}{ CED } & $\mathrm{Ah}_{1}$ & 10YR4/1 & 14.6 & 29.2 & 56.2 & nd & 0.8 & nd & nd & nd & 1 \\
\hline & $\mathrm{Ah}_{2}$ & 10YR4/1 & 15.9 & 34.6 & 49.5 & nd & 1.1 & nd & nd & nd & 1 \\
\hline
\end{tabular}

*Color de acuerdo a las tablas Munsell (2000)

$\mathrm{CO}=$ carbono orgánico; Fed = hierro extraido con oxalato-citrato-bicarbonato; Feo = hierro extrído con oxalato ácido; nd = no determinado

Ref $=$ Referencias: ${ }^{1}$ Este trabajo; ${ }^{2}$ Solleiro-Rebolledo et al., 2006; ${ }^{3}$ Rivera Uria et al., 2007; 
arcilla, siendo el horizonte $\mathrm{Bk}$ el que registra el máximo $(32.7 \%)$, lo que, dada la menor proporción que posee el horizonte C (26.9\%), demuestra que fue formada por pedogénesis. Los valores de Feo y Fed son similares, pero el cociente Feo/Fed (Tabla 2) sugiere que es el horizonte Ap el que muestra menor intemperismo. Un rasgo que caracteriza al suelo son las acumulaciones de carbonato de calcio que rellenan fisuras, poros y biporos (Cabadas-Báez, 2004). El paleosuelo que subyace al suelo moderno tiene una edad de 13358 años cal AP, lo que indica la edad máxima del Calcisol (McClung de Tapia et al., 2005).

\subsubsection{San Pablo (SNP)}

También se ubica en una posición con buen drenaje en la parte baja del valle; tiene un espesor de $0.30 \mathrm{~m}$ y una secuencia de horizontes Ap-C, y se ha clasificado como un Fluvisol con un desarrollo incipiente (Figura 2, Tabla 1). Sin embargo, muestra una alta cantidad de arcilla y valores muy bajos de Feo/Fed (Tabla 2), lo que, en principio, se asocia con una mayor pedogénesis. $\mathrm{Al}$ analizar sus propiedades morfológicas, se observa que tiene una estructura pobremente desarrollada, muy friable $\mathrm{y}$ el horizonte $\mathrm{C}$ aún conserva laminaciones, denotando su carácter aluvial (Rivera-Uria, 2008). Solleiro-Rebolledo et al. (2011) obtuvieron una edad de 520 años cal AP en un pedosedimento que subyace el Fluvisol, por lo que cabe suponer que el suelo SNP tiene una edad menor.

\subsubsection{Otumba (OTM)}

Se ubica en la parte baja del valle, en una posición geomórfica similar al de SNP, tiene un espesor de $0.7 \mathrm{~m}$ y una secuencia de horizontes $\mathrm{Ap}-\mathrm{AB}$ de color gris; está clasificado como un Fluvisol (Tabla 2). El horizonte Ap tiene estructura pobremente desarrollada, muy friable con abundantes raíces, con restos de cerámica en la superficie. El límite con $\mathrm{AB}$ es gradual y ondulado. El horizonte $\mathrm{AB}$ tiene una estructura en bloques subangulares, friable, pero más compacto. Un paleosuelo subyace al suelo moderno, y ha sido fechado en 3044 años cal AP (Cabadas-Báez, 2004).

\subsubsection{Cedazo (CED)}

Este perfil se ubica en la parte baja del valle y es un paleosuelo de $0.7 \mathrm{~m}$ de espesor, con horizontes Ap- $\mathrm{Ah}_{1-}$ $\mathrm{Ah}_{2}$, clasificado como un Vertisol. Este suelo corresponde a un paleosuelo sepultado en SNP a $1.25 \mathrm{~m}$ de profundidad, que aquí, por procesos de erosión, ha sido expuesto en la superficie y cuya edad es de 3166 años cal AP (SolleiroRebolledo et al., 2011). El horizonte superficial Ap posee un color pardo, tiene una estructura granular y relativamente friable. Es muy poroso, tiene abundantes raíces y el límite con el horizonte subyacente es claro y ondulado. Los horizontes $\mathrm{Ah}_{1}$ y $\mathrm{Ah}_{2}$ son de color negro, arcillosos (Tabla 2 ), con una estructura fuertemente desarrollada en bloques subangulares-angulares, muy compacta. Muestran grietas de pequeñas a medianas, las cuales son más pronunciadas $\mathrm{y}$ verticales en $\mathrm{Ah}_{2}$. El límite con el material subyacente, una roca andesítica, es abrupto.

\section{Metodología}

Para el análisis de propiedades magnéticas, se limpiaron secciones en los suelos, en las que se colectaron aproximadamente $400 \mathrm{~g}$ de suelo con un espaciamiento vertical de $5 \mathrm{~cm}$. Las muestras se secaron a una temperatura de $40{ }^{\circ} \mathrm{C}$ durante 24 horas y posteriormente fueron cuarteadas y conservadas dentro de un blindaje magnético para evitar que adquirieran una remanencia viscosa. Se llevó a cabo una serie de mediciones de parámetros magnéticos que permiten inferir la composición, concentración y distribución de los tamaños de partícula de los minerales magnéticos presentes en cada perfil de suelo.

Para determinar las principales fases magnéticas presentes en los suelos, en muestras de $\sim 1 \mathrm{~cm}^{3}$ se midió la susceptibilidad magnética volumétrica $(\kappa)$ en función de la temperatura con un equipo Bartington (MS2), un sensor (MS2W) y un horno (MS2WFP), entre $20^{\circ} \mathrm{C}$ y $650{ }^{\circ} \mathrm{C}$. La identificación de las fases magnéticas presentes se hizo con base en su temperatura de Curie (Collinson, 1983; Tarling, 1983; Dunlop y Özdemir, 1997). En muestras de $8 \mathrm{~cm}^{3}$ encapsuladas en cubos de acrílico inicialmente se midió la susceptibilidad magnética en un sistema Bartington MS2 y un sensor dual MS2B, que es expresada en términos de masa, susceptibilidad magnética de específica $(\chi)$, en frecuencias bajas $(0.47 \mathrm{kHz})$ y altas $(4.7 \mathrm{kHz})$. A partir de la $\chi$ medida en dos frecuencias, se calculó la susceptibilidad dependiente de la frecuencia $\chi_{\mathrm{df}} \%=\left[\left(\chi_{\text {baja frecuencia }}-\chi_{\text {alta frecuencia }}\right.\right.$ )$\left.\left./ \chi_{\text {baja frecuencia }}\right] * 100\right)$.

La magnetización remanente anhisterética (MRA) se obtuvo de la aplicación de un campo directo constante de $50 \mu \mathrm{T}$, sobreimpuesto a un campo alterno decreciente (entre 0 y $100 \times 10^{-3} \mathrm{~T}$ ), en un desmagnetizador Molspin. La magnetización remanente isotermal (MRI) se adquirió aplicando pulsos electromagnéticos con un magnetizador ASC Scientific, modelo IM-10, en campos directos e inversos entre $5 \times 10^{-3} \mathrm{~T}$ y $1 \mathrm{~T}$. Se consideró como magnetización remanente isotermal de saturación (MRIS ${ }_{1 \mathrm{~T}}$ ) a aquella adquirida en un campo directo de 1 tesla. Las remanencias artificiales se midieron con un magnetómetro Molspin Minispin Fluxgate, y a partir de estas remanencias se calcularon las coercitividades remanentes $\left(\mathrm{H}_{\mathrm{CR}}\right)$, los factores $\mathrm{S}_{300}\left(\mathrm{MRI}_{-300 \mathrm{mT}} / \mathrm{MRIS}_{1 \mathrm{~T}}\right)$ y la magnetización remanente isotermal adquirida en campos altos, $>300 \mathrm{mT}$, MRIA $_{300}=\left[\left(\right.\right.$ MRIS $\left.\left._{1 \mathrm{~T}}-\mathrm{MRI}_{-300 \mathrm{mT}}\right) / 2\right]$.

\section{Resultados}

\subsection{Curvas $\kappa v s . \mathrm{T}$}

En general las curvas de $\kappa v s$. T son reversibles en la mayoría de los perfiles y similares entre sí (Figura 3), 

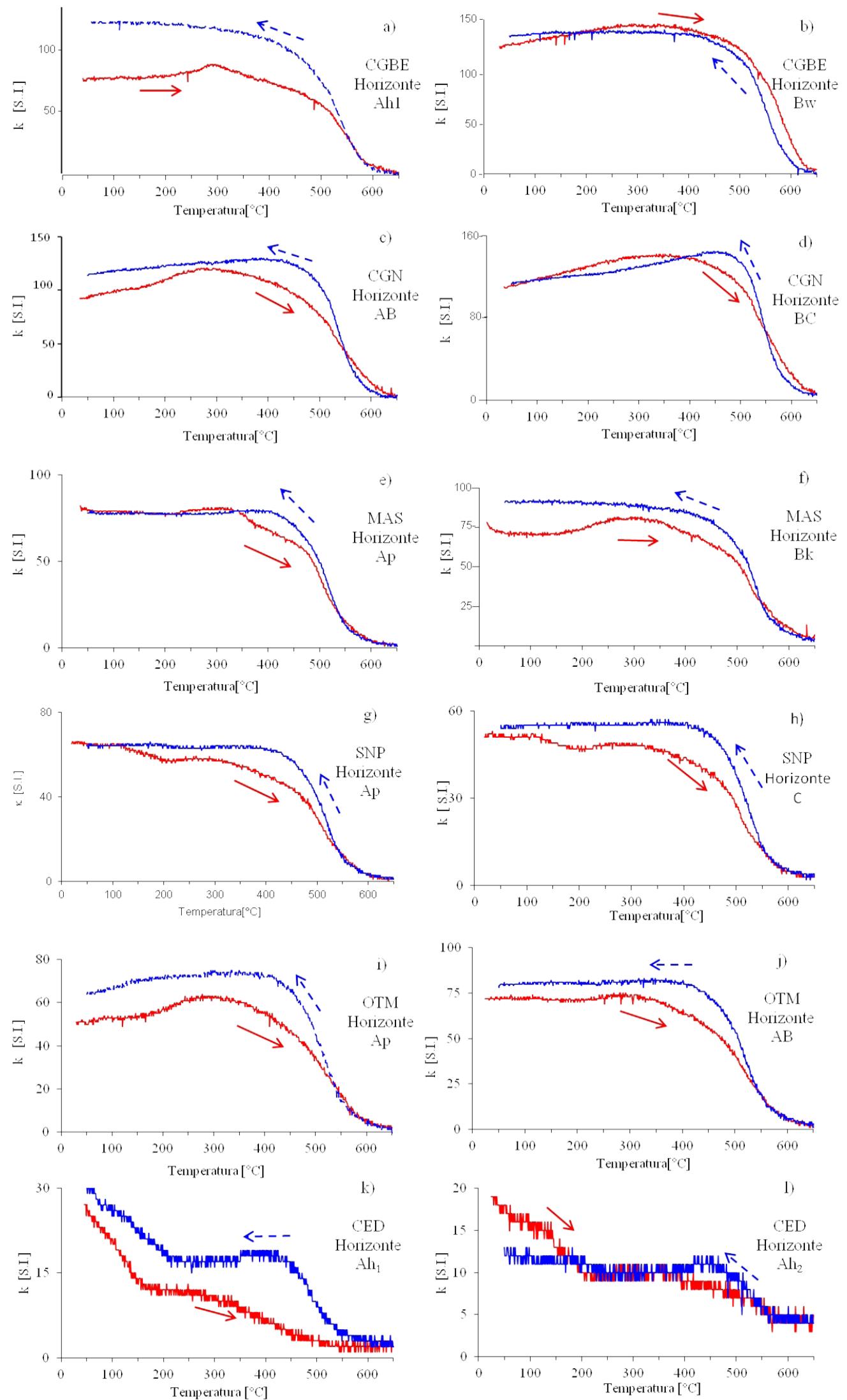

Figura 3. Curvas representativas de la susceptibilidad magnética en función de la temperatura $(\chi v s$. T $)$ utilizadas para identificar la mineralogía magnética presente en los suelos. La línea roja representa el calentamiento, mientras que la azul representa el enfriamiento. Horizontes Ah $(a)$ y Bw (b) de CGBE; horizontes AB (c) y BC (d) de CGN; horizontes Ap (e) y Bk (f) de MAS; horizontes Ap (g) y C (h) de SNP; horizontes Ap y AB de OTM; y horizontes $\mathrm{Ah}_{1}(\mathrm{k})$ y $\mathrm{Ah}_{2}(\mathrm{l})$ del perfil CED. 
presentando cambios en diferentes intervalos de temperatura. En el perfil CGBE las curvas de $\kappa v s$. T de las muestras de los horizontes superficiales Ah no son reversibles (Figura 3a) y presentan un claro incremento entre 200 y $300{ }^{\circ} \mathrm{C}$ durante el calentamiento, un suave decremento hasta 530 ${ }^{\circ} \mathrm{C}$ aproximadamente, y abrupto a partir de esa temperatura, mientras que las muestras de la parte baja del perfil (Bw) tienden a ser reversibles (Figura $3 b$ ). CGN tiene curvas $\kappa v s$. $\mathrm{T}$ que se caracterizan por la tendencia a incrementarse desde el inicio del calentamiento, hasta intervalos que oscilan entre 250 y $400{ }^{\circ} \mathrm{C}$, decreciendo posteriormente de forma suave y gradual, incluso por arriba de $600{ }^{\circ} \mathrm{C}$. Al igual que en CGBE, las muestras de los horizontes superficiales Ap y Ah (Figura 3c) poseen curvas irreversibles, mientras que en los horizontes inferiores $\mathrm{AB}$ y $\mathrm{BC}$, las curvas tienden a ser reversibles (Figura 3d).

Las curvas de $\kappa v s$. T del perfil MAS tienden a ser reversibles en la parte superficial del perfil (Figura 3e), zona donde se observa un ligero aumento en la curva durante el calentamiento que oscila entre 200 y $300{ }^{\circ} \mathrm{C}$. Posteriormente decrecen de forma suave hasta $500{ }^{\circ} \mathrm{C} \mathrm{y}$ de forma relativamente abrupta hasta el final del proceso, incluso por arriba de $600{ }^{\circ} \mathrm{C}$. Las muestras de ubicadas en la parte baja del perfil (horizontes $\mathrm{Bk}$ y C) presentan curvas menos reversibles (Figura 3f).

En SNP, las curvas de $\kappa$ vs. T son moderadamente reversibles, en especial en la parte superficial del suelo, donde decrecen entre 100 y $150{ }^{\circ} \mathrm{C}$ en la etapa de calentamiento, tienen un leve incremento entre 200 y $300{ }^{\circ} \mathrm{C}$ $\mathrm{y}$ decrecen de forma suave hasta el final del proceso (Figura $3 \mathrm{~g}$ ). En las muestras de la parte baja del perfil, el decremento es suave entre 300 y $500{ }^{\circ} \mathrm{C}$ y abrupto hacia el final del calentamiento (Figura 3h). En OTM, las curvas de $\kappa$ vs. T (Figuras 3i y 3j) son también moderadamente reversibles, similares a las registradas en SNP. Las curvas del horizonte Ap son las menos reversibles y se caracterizan por un incremento desde el inicio del experimento hasta $250{ }^{\circ} \mathrm{C}$, decrecen suavemente a partir de $300^{\circ} \mathrm{C}$, incluso por arriba de $600^{\circ} \mathrm{C}$ (Figura 3i). Durante el proceso de enfriamiento estas muestras son reversibles hasta $550{ }^{\circ} \mathrm{C}$ aproximadamente, siendo irreversible en el resto de esta etapa.

En el perfil CED, las curvas $\kappa v s$. T se caracterizan por el marcado decremento entre 50 y $200{ }^{\circ} \mathrm{C}$, un segundo decremento entre 350 y $380^{\circ} \mathrm{C}$ y entre 550 y $580^{\circ} \mathrm{C}$; a partir de estas temperaturas describen una trayectoria horizontal (Figuras 3k y 31). En la parte superior del suelo, las curvas tienden a ser menos reversibles (Figura 3k), en tanto que en la parte la parte baja del suelo, las curvas son reversibles (Figura 31).

\subsection{Parámetros magnéticos en función de la profundidad}

Las Figuras 4, 5, 6, 7, 8 y 9 muestran las curvas de los parámetros magnéticos analizados en los seis perfiles estudiados. Su posición en el relieve, diferencias climáticas y de vegetación, así como la edad de los materiales parentales han desarrollado suelos con diferentes grados de evolución, lo que se refleja en los parámetros magnéticos.

En el perfil de la cima del cerro Gordo (CGBE), las curvas de los parámetros de los horizontes de este suelo presentan claramente diferencias entre sí. Los perfiles de los parámetros dependientes de la concentración de minerales

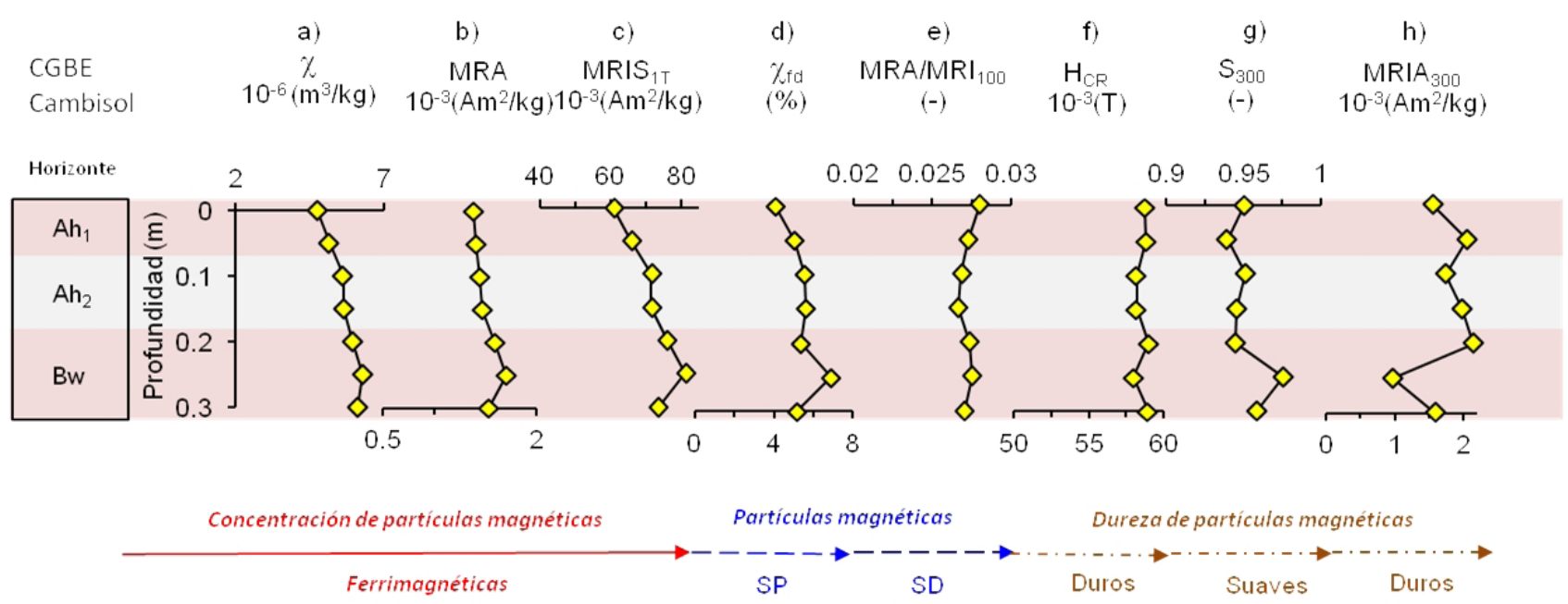

Figura 4. Parámetros magnéticos analizados del suelo CGBE. Las curvas que corresponden a los parámetros magnéticos asociados con la concentración de minerales magnéticos son: a) Susceptibilidad magnética, $\chi$, (concentración de minerales magnéticos); b) Magnetización remanente anhisterética MRA (concentración de ferrimagnéticos, sensible a la abundancia de partículas de dominio sencillo DS, $<0.1 \mu \mathrm{m}$ ); y c) Magnetización remanente isotermal

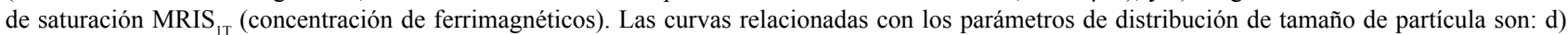
Susceptibilidad magnética dependiente de la frecuencia $\chi_{\mathrm{fd}} \%$ (partículas SP, $<0.01 \mu \mathrm{m}$ ); y e) Cociente MRA/MRI ${ }_{100}($ abundancia de partículas DS). Los parámetros magnéticos asociados con la "dureza" de las partículas magnéticas son: $\mathrm{f}$ ) Coercitividad remanente $\mathrm{H}_{\mathrm{CR}}$ (sensible a la presencia de partículas "duras", hematita, goethita y magnetita DS); g) Cociente S isotermal de saturación en campos altos $(>300 \mathrm{mT}), \mathrm{MRIA}_{300}$ (concentración de hematita o goethita). 


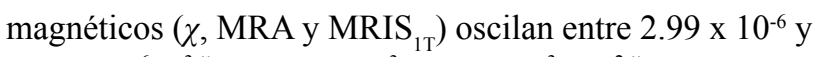
$6.47 \times 10^{-6} \mathrm{~m}^{3} / \mathrm{kg} ; 0.85 \times 10^{-3}$ y $1.7 \times 10^{-3} \mathrm{Am}^{2} / \mathrm{kg} ;$ y $62.73 \mathrm{x}$ $10^{-3}$ y $79.32 \times 10^{-3} \mathrm{Am}^{2} / \mathrm{kg}$, respectivamente. Se caracterizan por mostrar los valores más altos en el horizonte $\mathrm{Bw}$ y una disminución hacia la parte superficial, alcanzando en la cima del horizonte $\mathrm{Ah}_{1}$ los más bajos (Figuras $4 \mathrm{a}, 4 \mathrm{~b}$ y $4 \mathrm{c}$ ). Los parámetros $\chi_{\mathrm{fd}} \%$ y MRA/MRI 100 (dependientes de tamaños de dominio pequeños) varían entre 2.5 y $6.9 \%$, y entre 0.027 y 0.028 , respectivamente. El parámetro $\chi_{\mathrm{fd}} \%$ registra un aumento moderado en el horizonte $\mathrm{Bw}$ y decrece en los horizontes A (Figura 4d). El cociente MRA/MRI también muestra un ligero aumento tanto en el horizonte $\mathrm{Bw}$ como en el $\mathrm{Ah}_{1}$ (Figura 4e). Los parámetros relacionados con la dureza de las partículas magnéticas $\left(\mathrm{H}_{\mathrm{CR}}, \mathrm{S}_{300}\right.$ y MRIA $\left.{ }_{300}\right)$ presentan únicamente ligeras variaciones a lo largo del perfil, y sus valores oscilan entre $58 \times 10^{-3}$ y $59 \times 10^{-3} \mathrm{~T} ; 0.93$ y $0.97 \%$; 0.96 x $10^{-3}$ y $2.13 \times 10^{-3} \mathrm{Am}^{2} / \mathrm{kg}$, respectivamente (Figuras 4f, 4g y $4 \mathrm{~h}$ ).

En el perfil ubicado en la ladera de cerro Gordo (CGN), las curvas de los parámetros analizados también presentan contrastes entre los diferentes horizontes: $\chi\left(2.99 \times 10^{-6} \mathrm{a}\right.$ $\left.5.82 \times 10^{-6} \mathrm{~m}^{3} / \mathrm{kg}\right)$, MRA $\left(0.85 \times 10^{-3}\right.$ a $\left.1.71 \times 10^{-3} \mathrm{Am}^{2} / \mathrm{kg}\right)$

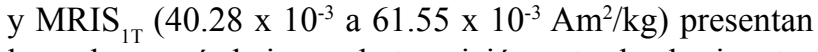
los valores más bajos en la transición entre los horizontes $\mathrm{BC}$ y $\mathrm{AB}$ (de 0.50 a $0.45 \mathrm{~m}$ de profundidad), mientras que en los horizontes Ah y Ap se registran los valores más altos (Figuras $5 \mathrm{a}, 5 \mathrm{~b}$ y $5 \mathrm{c}$ ). Los parámetros $\chi_{\mathrm{fd}} \%$ y MRA/
MRI $_{100}$ oscilan entre 2.5 y $5.1 \%$ y entre 0.025 y 0.041 , respectivamente. En el horizonte $\mathrm{BC}$ se presentan los valores más bajos de ambos parámetros, en tanto que en los horizontes $\mathrm{AB}$ y Ah existe un aumento moderado (más marcado en MRA/MRI ${ }_{100}$ ), que representa los mayores valores encontrados en estos parámetros (Figuras $5 \mathrm{~d}$ y $5 \mathrm{e}$ ). Los parámetros de dureza magnética (Figuras $5 \mathrm{f}, 5 \mathrm{~g}$ y $5 \mathrm{~h}$ ) presentan un comportamiento relativamente constante, varían entre $55 \times 10^{-3}$ y $56 \times 10^{-3} \mathrm{~T}\left(\mathrm{H}_{\mathrm{CR}}\right) ; 0.94$ a $0.96 \%\left(\mathrm{~S}_{300}\right)$ y $0.93 \times 10^{-3}$ a $1.67 \times 10^{-3} \mathrm{Am}^{2} / \mathrm{kg}\left(\mathrm{MRIA}_{300}\right)$. Las mayores variaciones están en MRIA $_{300}$, con los valores más altos en la parte baja de BC y en Ap.

En el perfil MAS los parámetros magnéticos presentan ligeras variaciones verticales: $\chi$ varía entre $2.65 \times 10^{-6} \mathrm{y}$ $5.53 \times 10^{-6} \mathrm{~m}^{3} / \mathrm{kg}$; MRA entre $0.60 \times 10^{-3}$ y $1.01 \times 10^{-3}$ $\mathrm{Am}^{2} / \mathrm{kg}$; y $\mathrm{MRIS}_{1 \mathrm{~T}}$ entre 20.77 x $10^{-3}$ y $27.04 \times 10^{-3} \mathrm{Am}^{2} /$ $\mathrm{kg}$. La más notoria de estas variaciones es el aumento en $\chi$ en la parte superior del horizonte Bk (Figura 6a), mismo que no se refleja en MRA ni MRIS ${ }_{1 \mathrm{~T}}$. Los parámetros $\chi_{\mathrm{fd}} \%$ y MRA/MRI ${ }_{100}$ oscilan entre 2.6 y $6.9 \%$ y entre 0.030 y 0.051, respectivamente. Ambos parámetros tienen valores moderadamente altos en el horizonte $\mathrm{C}$ y en la parte baja de $\mathrm{Bk}$, mientras que en la parte superior de $\mathrm{Bk}$ y en el horizonte Ap presentan sus menores valores (Figuras $6 \mathrm{~d}$ y $6 \mathrm{e}$ ). Los parámetros $\mathrm{H}_{\mathrm{CR}}, \mathrm{S}_{300}$ y MRIA $_{300}$ muestran valores constantes que oscilan entre $54 \times 10^{-3}$ y $56 \times 10^{-3} \mathrm{~T}\left(\mathrm{H}_{\mathrm{CR}}\right) ; 0.93$ y 0.97 $\%\left(\mathrm{~S}_{300}\right)$; y $0.307 \times 10^{-3}$ y $0.795 \times 10^{-3} \mathrm{Am}^{2} / \mathrm{kg}\left(\mathrm{MRIA}_{300}\right)$,

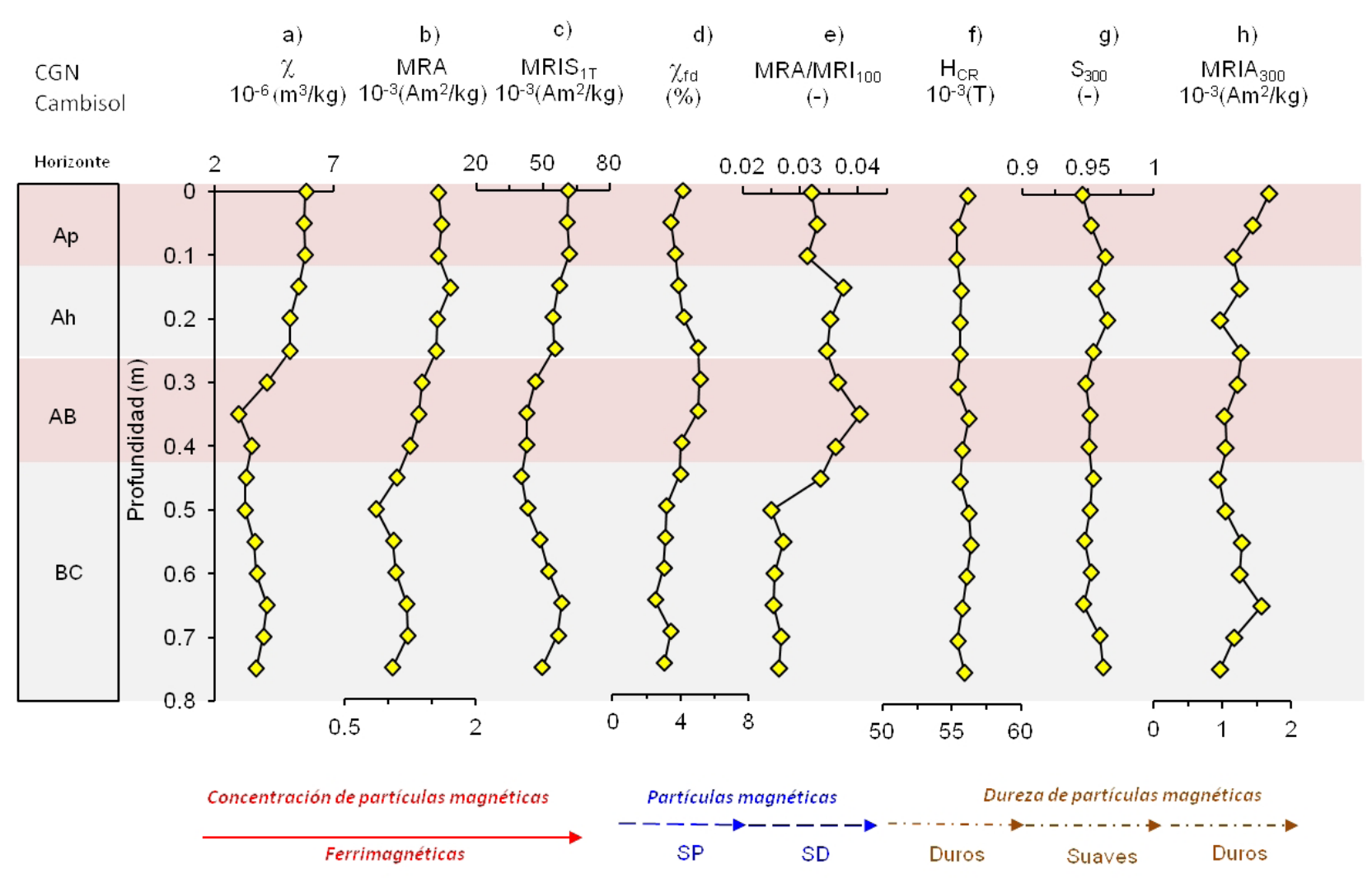

Figura 5. Parámetros magnéticos analizados para el perfil CGN. (Ver pie de Figura 4). 


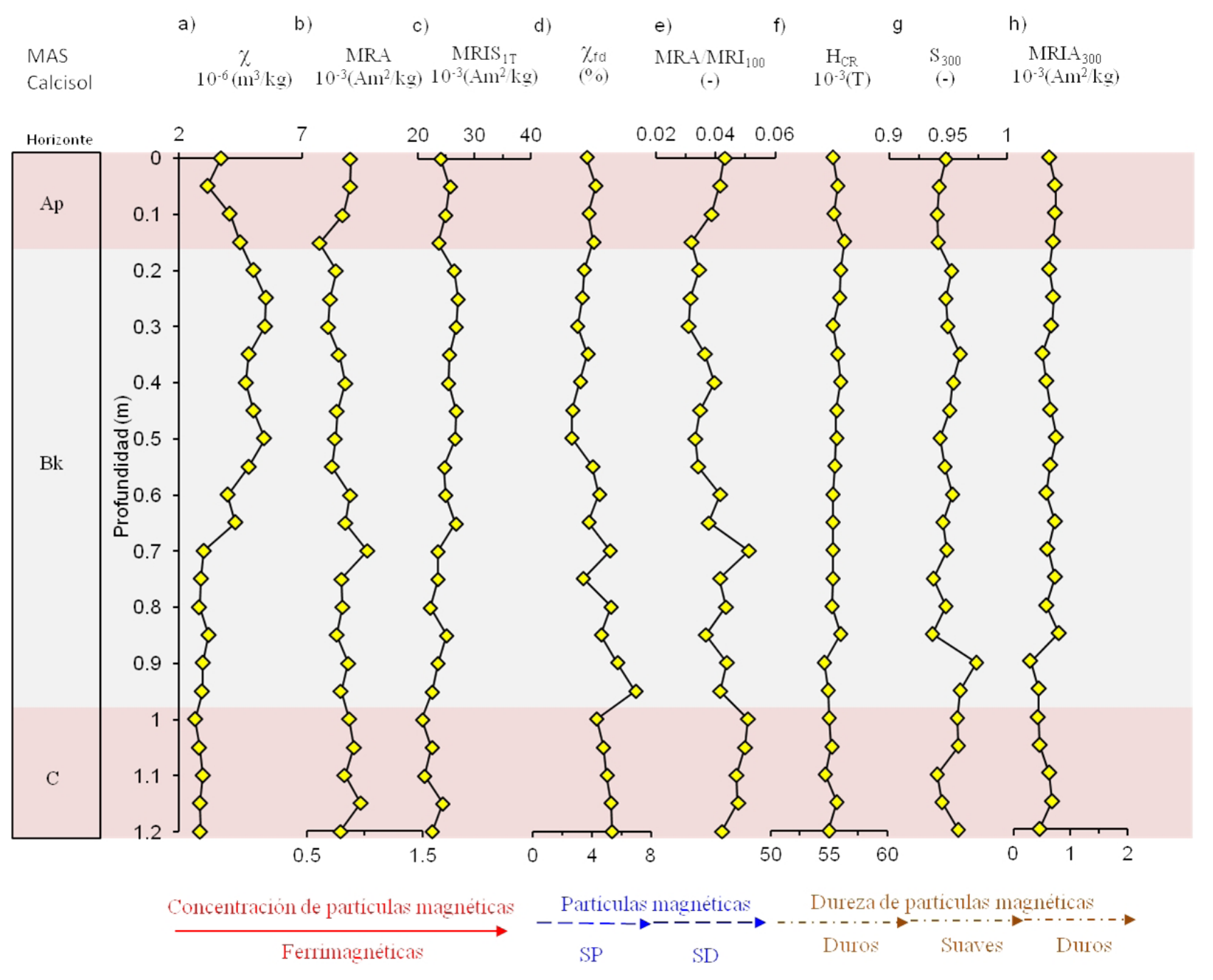

Figura 6. Parámetros magnéticos analizados para el perfil MAS. (Ver pie de Figura 4).

sin variaciones significativas a lo largo del perfil.

En el perfil San Pablo (SNP) los parámetros magnéticos analizados se caracterizan por tener curvas con variaciones relativamente pequeñas. Los parámetros de concentración magnética (Figuras $7 \mathrm{a}, 7 \mathrm{~b}$ y $7 \mathrm{c}$ ) oscilan entre $2.78 \times 10^{-6} \mathrm{y}$ $3.17 \times 10^{-6} \mathrm{~m}^{3} / \mathrm{kg}(\chi) ; 0.555 \times 10^{-3}$ y $0.627 \times 10^{-3} \mathrm{Am}^{2} / \mathrm{kg}$ (MRA); y $21.76 \times 10^{-3}$ y $24.34 \times 10^{-3} \mathrm{Am}^{2} / \mathrm{kg}\left(\mathrm{MRIS}_{1 \mathrm{~T}}\right) \cdot \chi_{\mathrm{fd}} \%$ varía entre 3 y $5 \%$, cuyo valor más alto se presenta en la parte superior del horizonte Ap. MRA/MRI ${ }_{100}$, cuyos valores varían entre 0.032 y 0.037 , describe una trayectoria similar $\mathrm{a} \chi_{\mathrm{fd}} \%$ (Figura $7 \mathrm{~d}$ ), mientras que $\mathrm{H}_{\mathrm{CR}}\left(\right.$ de $58 \times 10^{-3} \mathrm{a} 61 \times 10^{-3}$ T), $\mathrm{S}_{300}$ (de 0.90 a $0.94 \%$ ) y MRIA $_{300}$ (de 0.6121 x $10^{-3}$ a 1.13 x $10^{-3} \mathrm{Am}^{2} / \mathrm{kg}$ ) no registran cambios significativos, salvo la ocurrencia de mayores coercitividades $\left(\mathrm{S}_{300}\right.$ y MRIA $\left._{300}\right)$ en la parte baja del horizonte $\mathrm{C}$ (Figuras $7 \mathrm{e}, 7 \mathrm{f}, 7 \mathrm{~g}$ y $7 \mathrm{~h}$ ).

En Otumba (OTM) las variaciones en los parámetros analizados son sutiles, $\left(\chi\right.$, de $2.43 \times 10^{-6}$ a $3.40 \times 10^{-6} \mathrm{~m}^{3} /$ $\mathrm{kg}$; MRA, de $0.579 \times 10^{-3}$ a $1.10 \times 10^{-3} \mathrm{Am}^{2} / \mathrm{kg}$; y $\mathrm{MIRS}_{1 \mathrm{~T}}$, de $23.31 \times 10^{-3}$ a $28.30 \times 10^{-3} \mathrm{Am}^{2} / \mathrm{kg}$ ). En general, en el horizonte $\mathrm{AB}$ el comportamiento de los parámetros es casi constante, con ligeras variaciones en la transición al horizonte Ap. Los parámetros $\chi$ y MRIS $_{1 \mathrm{~T}}$ muestran un ligero aumento en el horizonte Ap con relación al horizonte subyacente $\mathrm{AB}$ (Figuras $8 \mathrm{a}$ y $8 \mathrm{c}$ ). La mayor diferencia está en los perfiles $\chi_{\mathrm{fd}} \%$ (de 4 a $26 \%$ ) y MRA/MRI ${ }_{100}$ (de 0.027 a 0.048 ), que son mayores en $A B$ y disminuyen en Ap (Figuras 8 f y $8 \mathrm{~g}$ ). De manera correspondiente, los parámetros $\mathrm{H}_{\mathrm{CR}}$ (de $44.5 \times 10^{-3}$ a $46 \times 10^{-3} \mathrm{~T}$ ), $\mathrm{S}_{300}$ (de 0.92 a $0.94 \%$ ) y MRIA $_{300}$ (de $0.683 \times 10^{-3} \mathrm{a} 1.07 \times 10^{-3} \mathrm{Am}^{2} / \mathrm{kg}$ ) presentan en general la mayor coercitividad en el horizonte Ap (Figuras $8 \mathrm{~h} \mathrm{y} 8 \mathrm{j}$ ).

Los gráficos de los parámetros magnéticos en el perfil Cedazo (CED) en su mayor parte muestran variaciones sistemáticas entre los horizontes que lo constituyen. Los parámetros de concentración oscilan entre $0.86 \times 10^{-6} \mathrm{y}$ $5.15 \times 10^{-6} \mathrm{~m}^{3} / \mathrm{kg}(\chi) ; 0.107 \times 10^{-3}$ y $0.307 \times 10^{-3} \mathrm{Am}^{2} /$ kg (MRA); y 3.94 x $10^{-3}$ y $33.01 \times 10^{-3} \mathrm{Am}^{2} / \mathrm{kg}\left(\right.$ MRIS $_{1 \mathrm{~T}}$ ). Éstos presentan los valores más bajos en el horizonte $\mathrm{Ah}_{2} \mathrm{y}$ la parte baja del $\mathrm{Ah}_{1}$ (entre 0.7 y $0.3 \mathrm{~m}$ de profundidad), y a 


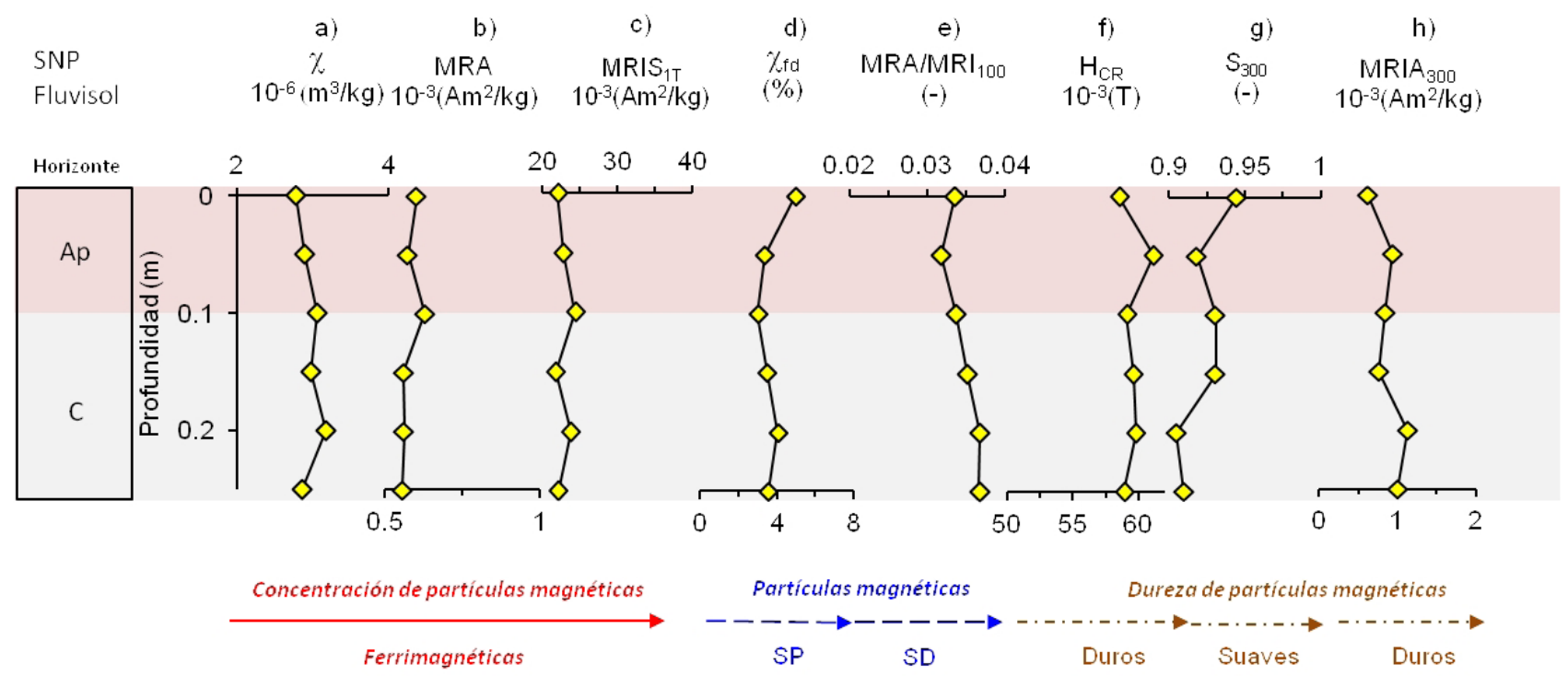

Figura 7. Parámetros magnéticos analizados para el perfil SNP. (Ver pie de Figura 4).
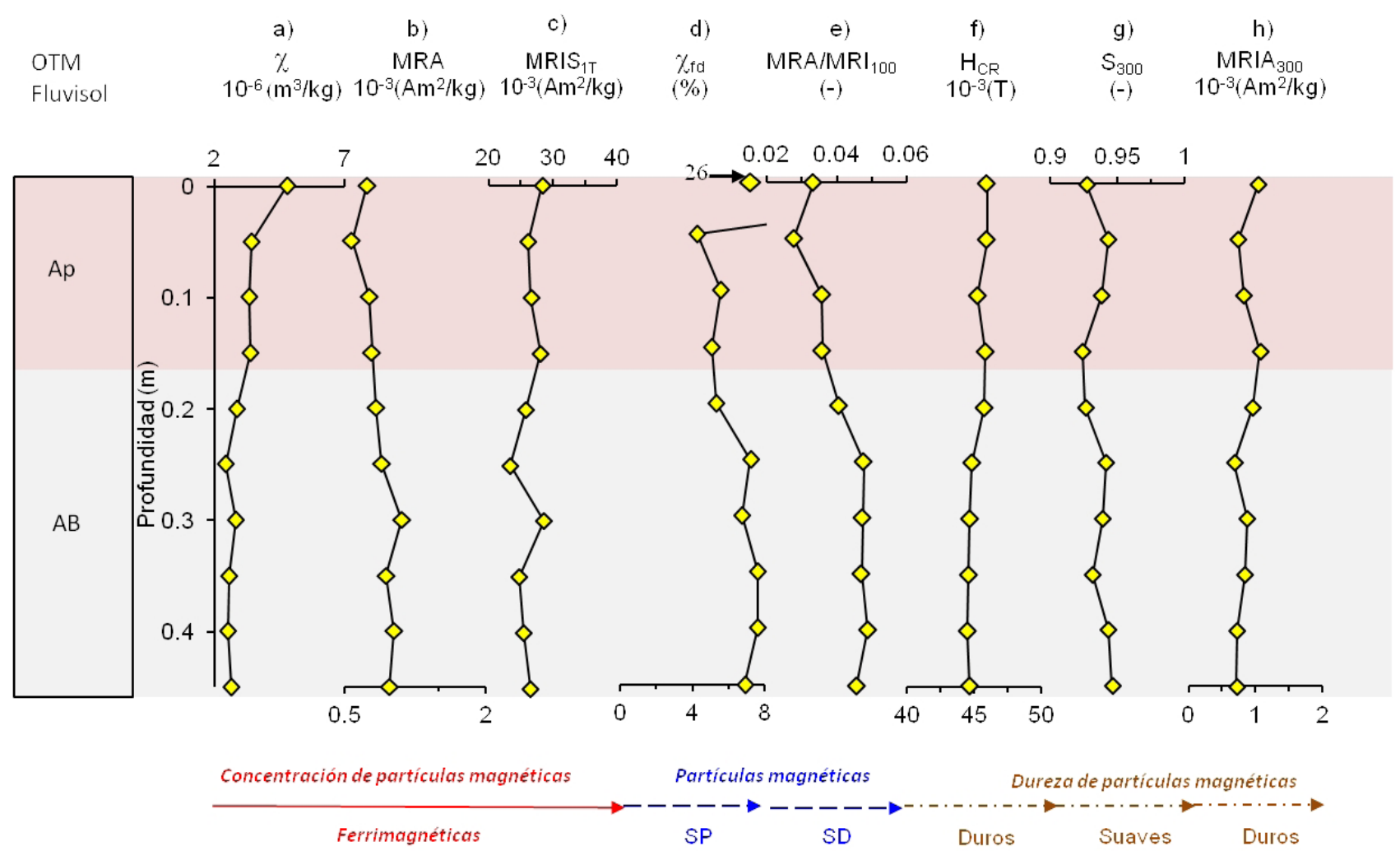

Figura 8. Parámetros magnéticos analizados para el perfil OTM. (Ver pie de Figura 4).

partir de ese nivel aumentan hasta la superficie (Figuras 9a, $9 \mathrm{~b}$ y $9 \mathrm{c})$. Los parámetros $\chi_{\mathrm{fd}} \%$ y MRA/MRI ${ }_{100}$ varían entre 0.2 y $4 \%$ y 0.014 y 0.040 , respectivamente. El incremento en los parámetros de concentración en la parte superior del perfil coincide con la disminución en $\chi_{\mathrm{fd}} \%$ y MRA/MRI ${ }_{100}$ (Figuras $9 \mathrm{~d}$ y $9 \mathrm{e}$ ). Los parámetros de coercitividades son relativamente constantes a los largo del perfil, oscilan entre 60 x $10^{-3}$ y $78 \times 10^{-3} \mathrm{~T}\left(\mathrm{H}_{\mathrm{CR}}\right) ; 0.85$ y $0.97 \%\left(\mathrm{~S}_{300}\right) ; \mathrm{y} 0.075 \mathrm{x}$ $10^{-3}$ y $1.474 \times 10^{-3} \mathrm{Am}^{2} / \mathrm{kg}\left(\right.$ MRIA $\left._{300}\right)$, aunque las pequeñas diferencias no son consistentes entre sí. En la transición entre los horizontes $\mathrm{Ah}_{1}$ y Ap hay un ligero aumento en la coercitividad (Figuras 9f y 9g). 


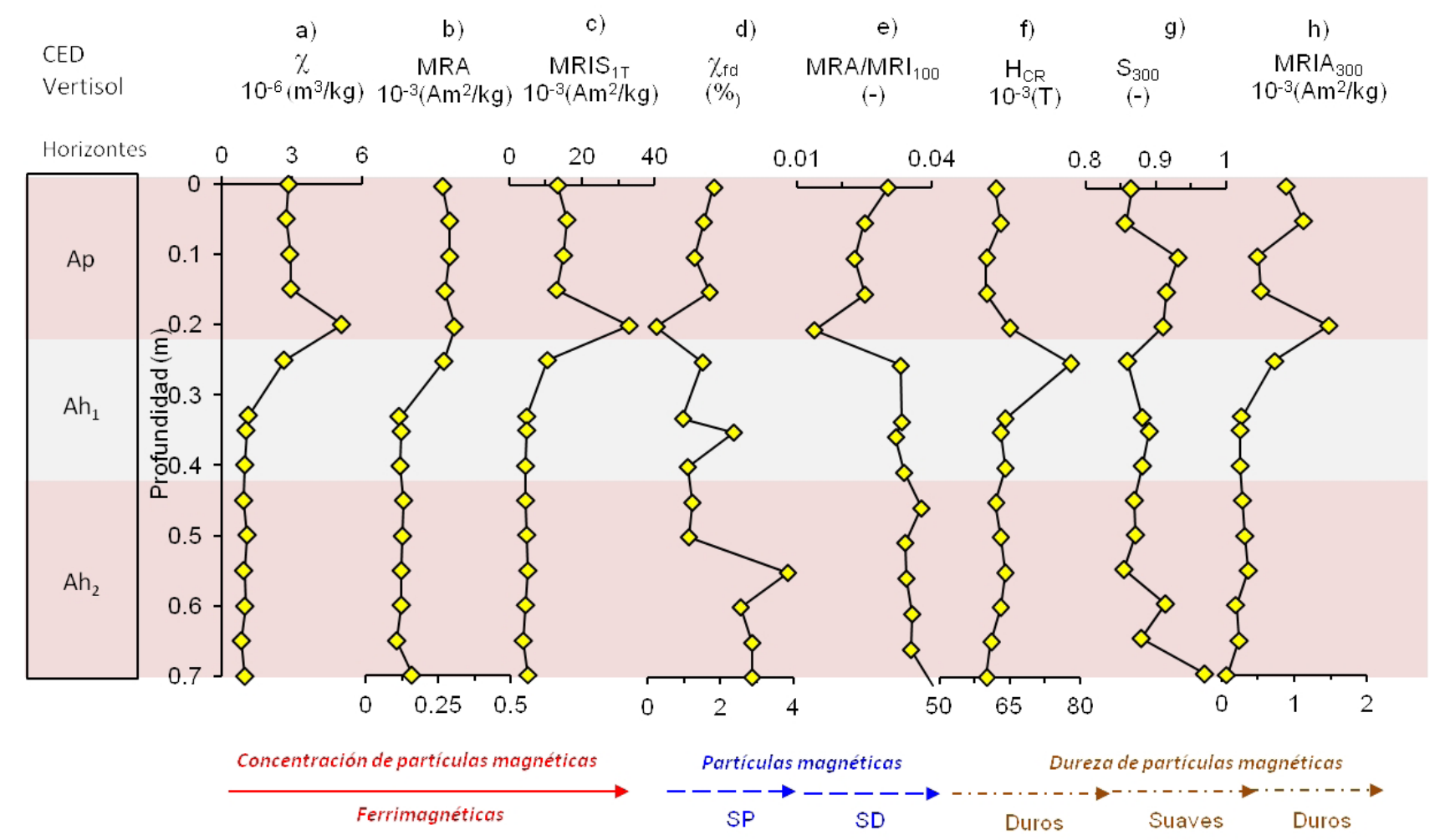

Figura 9. Parámetros magnéticos analizados para el perfil CED. (Ver pie de Figura 4).

\section{Discusión}

\subsection{Interpretación de parámetros magnéticos}

La $\chi$ es la suma de la contribución de minerales tanto ferrimagnéticos como antiferrimagnéticos y paramagnéticos (ferromagnesianos en general), en tanto que los parámetros MRA y MRIS ${ }_{1 \mathrm{~T}}$ lo son sólo de las partículas ferrimagnéticas. La medición de la susceptibilidad magnética $(\kappa)$ durante el calentamiento permite la identificación directa de los minerales magnéticos presentes a través de la temperatura de Curie. Esta temperatura es de $580{ }^{\circ} \mathrm{C}$ para la magnetita pura, entre 597 y $657^{\circ} \mathrm{C}$ para la maghemita, $675^{\circ} \mathrm{C}$ para la hematita y entre 80 y $150{ }^{\circ} \mathrm{C}$ para la goethita (Morin et al., 1950; Hunt et al., 1995; Özdemir et al., 1993; Özdemir y Dunlop, 1996). El contenido de Ti en magnetita y maghemita decrece la temperatura de Curie (Miyahara, 1972; Aragón et al., 1985). Sin embargo, la información que brindan las curvas $\kappa v s$. T es en ocasiones incompleta debido a que en estos análisis, por una parte, las fases ferrimagnéticas (Ti-magnetita y Ti-maghemita) dominan sobre las antiferrimagnéticas (hematita y goethita) y, por la otra, durante el calentamiento ocurren una serie de transformaciones en los minerales magnéticos, lo que es la causa más probable de la irreversibilidad de las curvas durante el enfriamiento (Dunlop y Özdemir, 1997). Por ello, la información obtenida con las mediciones $\kappa v s$. $\mathrm{T}$ se complementa con otros indicadores de mineralogía magnética basados en la coercitividad $\left(\mathrm{H}_{\mathrm{CR}}\right.$, cocientes $\mathrm{S}_{300} \mathrm{y}$ MRIA $_{300}$ ), los cuales discriminan las fases magnéticas duras (hematita y goethita) que están pobremente representadas en los análisis $\kappa v s$. T. La coercitividad remanente $\mathrm{H}_{\mathrm{CR}}$ (el valor del campo inverso necesario para convertir una magnetización de saturación en cero remanencia) depende no sólo de la mineralogía (mayores valores de $\mathrm{H}_{\mathrm{CR}}$ en hematita y goethita), sino del tamaño de partícula, ya que los granos finos DS tienen mayor coercitividad. El cociente $\mathrm{S}_{300}$ es interpretado como un indicador de la abundancia relativa de minerales magnéticos duros, en el cual valores menores indican mayor abundancia de hematita o goethita, mientras que valores altos de $\mathrm{MRIA}_{300}$ son un indicador de la concentración de los mismos (Opdike y Channell, 1996).

Los cocientes indicadores de tamaño de partícula son $\chi_{\mathrm{fd}} \%$ y MRA $/ \mathrm{MRI}_{100} \cdot \chi_{\mathrm{fd}} \%$ es indicador de la existencia de partículas ultrafinas superparamagnéticas (SP, $<0.01 \mu \mathrm{m})$, en el que $\chi_{\mathrm{fd}} \%<2 \%$ implica que la abundancia de partículas SP es menor al $10 \% ; \chi_{\mathrm{fd}} \% \sim 8 \%$ indica un contenido de SP $>75 \%$; y $\chi_{\mathrm{fd}} \%$ con valores entre 10 y $14 \%$ indica la predominancia partículas SP(Dearing, 1994; Dearing et al., 1997). El cociente de la MRA normalizada por la MRI obtenida en un campo de $100 \times 10^{-3} \mathrm{~T}$ (MRA/MRI ${ }_{100}$ ) es utilizado para estimar la abundancia relativa de partículas DS (Hunt et al., 1995). Los diagramas de dispersión que combinan la información obtenida a través de los parámetros 
magnéticos, permiten comparar las características de las propiedades magnéticas de todos los perfiles de suelos en conjunto.

\subsection{Propiedades magnéticas de los perfiles}

De acuerdo a las curvas $\kappa v s$. T, a lo largo del perfil CGBE (Cambisol) la fase dominante es Ti-magnetita, que en los horizontes superficiales coexiste con Ti-maghemita y con hematita (Figura 3a). En el horizonte Bw hay una proporción menor de hematita (Figura 3b). Ésta no se refleja en las curvas $\mathrm{S}_{300} \mathrm{y} \mathrm{H}_{\mathrm{CR}}$, aunque MRIA M $_{300}$ es ligeramente alto (Figuras 4f, 4g y 4h). La continua disminución en la concentración magnética hacia la parte superficial del suelo (Figuras 4a, 4b y 4c) coincide con un decremento en la cantidad de partículas SP, y aunque también hay un leve decremento en el contenido de partículas DS, éstas aumentan levemente en el horizonte $\mathrm{Ah}_{1}$. Estas características sugieren que en el perfil CGBE ocurre una destrucción gradual de la mineralogía magnética primaria, Ti-magnetita y Ti-maghemita, hacia la cima del mismo, lo que resulta también en el engrosamiento del tamaño de partícula. En el horizonte $\mathrm{Ah}_{1}$, el leve incremento de partículas DS es posible que resulte de la destrucción parcial de partículas MD.

Las curvas $\kappa v s$. T de CGN (Cambisol) sugieren que las Ti-maghemita y Ti-magnetita son las fases mineralógicas más abundantes, y en menor proporción está presente hematita en todo el perfil (Figuras 3c y 3d). Al igual que en CGBE, los parámetros de coercitividad no muestran una contribución importante de hematita (Figuras $5 \mathrm{f}, 5 \mathrm{~g}$ y $5 \mathrm{~h}$ ), por lo que sus variaciones reflejan mayormente fluctuaciones en el tamaño de partículas. Únicamente hay un leve aumento en el contenido de hematita en el horizonte Ap. En CGN, el cambio más notable en las propiedades magnéticas es el aumento en la concentración en los tres horizontes superiores, especialmente Ah y Ap (Figuras $5 \mathrm{a}, 5 \mathrm{~b}$ y $5 \mathrm{c}$ ). Este aumento coincide con el incremento de partículas finas SP y DS (Figuras $5 \mathrm{~d}$ y $5 \mathrm{e}$ ), particularmente en los horizontes $\mathrm{AB}$ y Ah. La falta de simultaneidad en el aumento entre los parámetros dependientes de la concentración se explica porque éstos responden a tamaños específicos de partícula o de mineralogía. De acuerdo a esto, el horizonte BC tiene baja concentración ferrimagnética dominada por partículas gruesas tales como Ti-magnetita $\mathrm{MD}$, en los horizontes $\mathrm{AB}$ y Ah hay un aumento de Ti-magnetita de tamaño SP y DS, mientras que en el horizonte Ap hay un engrosamiento en el tamaño de partícula (> MD) y un leve aumento de hematita.

En MAS (Calcisol) las curvas $\kappa v s$. T registran en todo el perfil mezclas de minerales magnéticos que son controladas por fases de Ti-magnetita y que coexisten con Ti-maghemita y en menor proporción con hematita, misma que no se registra en las muestras analizadas en el horizonte Bk (Figura 3f). El contenido de minerales que contribuyen a MRIA ${ }_{300}$ (hematita y goethita) es de los más bajos registrados en todos los perfiles analizados. Los parámetros $\mathrm{H}_{\mathrm{CR}}, \mathrm{S}_{300}$ y MRIA ${ }_{300}$ muestran valores constantes, lo que indica que no hay variaciones significativas en la composición de los minerales magnéticos a lo largo del perfil (Figuras $6 \mathrm{f}, 6 \mathrm{~g}$ y $6 \mathrm{~h}$ ). En la parte superior del horizonte Bk, $\chi$ aumenta ligeramente, no así MRA ni MRIS $_{1 \mathrm{~T}}$, lo que sugiere que este incremento se debe a la presencia de minerales paramagnéticos que contribuyen a $\chi$, pero no a la remanencia (Figuras 6a, 6b y 6c). Los valores moderadamente altos en $\chi_{\mathrm{fd}} \%$ y MRA/MRI ${ }_{100}$ en el horizonte $\mathrm{C}$ y la parte baja de $\mathrm{Bk}$ indican una mezcla de tamaños de partículas SP-DS-MD en estas porciones, en tanto que en la parte superior de Bk y el horizonte Ap hay un ligero engrosamiento de las partículas y los tamaños cambian a una asociación de tamaños MD con cantidades menores de DS.

En SNP (Fluvisol) las curvas $\kappa v s$. T señalan mezclas relativamente más homogéneas en composición, en las cuales la Ti-magnetita coexiste con las fases de Ti-maghemita. El suave decaimiento entre 100 y $150^{\circ} \mathrm{C}$ sugiere la presencia de goethita (Figuras $3 g$ y $3 \mathrm{~h}$ ). Sólo el horizonte Ap presenta un decaimiento en $\kappa$ arriba de $580^{\circ} \mathrm{C}$, lo que indica la presencia de hematita. La presencia de fases magnéticamente duras (hematia y goethtita) no tienen una franca expresión en los indicadores de coercitividad, únicamente se reflejan tenuemente en el incremento en $\mathrm{MRIA}_{300} \mathrm{y}$ el decremento en $\mathrm{S}_{300}$ en la parte baja del horizonte C (Figura 7). En SNP los parámetros magnéticos presentan variaciones relativamente pequeñas que no permiten establecer de forma clara diferencias en el comportamiento de los mismos, en especial los parámetros de concentración magnética. Los valores de $\chi$, MRA y MRIS $_{1 \mathrm{~T}}$ indica una escasa presencia de minerales ferrimagnéticos (Figuras 7a, 7b y 7c). La variación en los indicadores de tamaño de partícula es mínima y los valores relativamente bajos de $\chi_{\mathrm{fd}} \%$ y MRA/ $\mathrm{MRI}_{100}$ sugieren dominancia de tamaños MD con cantidades menores de DS y SP.

Las muestras analizadas por $\kappa v s$. T del perfil OTM (Fluvisol) registran una mezcla de Ti-maghemita y Timagnetita, que coexisten con hematita (Figuras $3 \mathrm{i}$ y 3j). La coercitividad, observada en los parámetros $H_{C R}$, $\mathrm{S}_{300}$ y MRIA $_{300}$, es levemente mayor en la transición de horizontes AB-Ap y en la parte más superficial del perfil, pero no indican cantidades abundantes de hematita (Figura 8). Las variaciones en los perfiles de los parámetros analizados son sutiles pero, en general, en el horizonte $\mathrm{AB}$ la concentración ferrimagnética, reflejada en $\chi$ y MRIS, es baja y se incrementa en el horizonte Ap, alcanzando su valor más alto en la superficie del perfil. Los parámetros $\chi_{\mathrm{fd}} \%$ y MRA/MRI ${ }_{100}$ en el horizonte $\mathrm{AB}$ sugieren mezclas con un contenido cercano al $75 \%$ de partículas SP y un aumento de partículas DS, mismas que disminuyen en Ap, lo que sugiere un engrosamiento del tamaño de partícula en ese horizonte. Únicamente en la parte superficial de Ap dominan las partículas SP (> $75 \%)$.

En los horizontes $\mathrm{Ah}_{1}$ y Ap de CED, el súbito decremento en $\kappa v s$. T entre 50 y $150{ }^{\circ} \mathrm{C}$ sugiere la presencia de Ti-magnetita o Ti-maghemita con alto contenido de $\mathrm{Ti}$ 
(Figura 3k), en tanto que en el horizonte $\mathrm{Ah}_{2}$ el contenido de Ti es menor. En este horizonte, el cambio de pendiente en la curva $\kappa v s$. T alrededor de los $150{ }^{\circ} \mathrm{C}$ (Figura 31), sugiere la presencia de goethita. Los parámetros $\mathrm{H}_{\mathrm{CR}}$ y $\mathrm{S}_{300}$ presentan los valores más altos y más bajos, respectivamente, de todos los suelos analizados, lo que apoya la existencia de minerales antiferrimagnéticos en el perfil (Figuras 9f y 9g). En los horizontes $\mathrm{Ah}_{2}$ y $\mathrm{Ah}_{1}$, MRIA $_{300}$ indica bajas concentraciones de estos minerales, por lo que los relativamente bajos valores de $\mathrm{S}_{300}$ y altos valores de $\mathrm{H}_{\mathrm{CR}}$ en ellos también pueden indicar partículas ferrimagnéticas de mayor dureza (DS). CED presenta en los horizontes $\mathrm{Ah}_{2}$ y $\mathrm{Ah}_{1}$ las menores concentraciones de minerales ferrimagnéticos encontradas en este estudio. En la parte superior del perfil, a partir de la transición entre los horizontes $\mathrm{Ah}$ y Ap, hay un enriquecimiento tanto de partículas ferrimagnéticas como de antiferrimagnéticas, caracterizado por un aumento en el tamaño de partícula, $\operatorname{MD}\left(<\mathrm{MRA} / \mathrm{MRI}_{100}\right)$, y nulo contenido de $\operatorname{SP}\left(\chi_{\mathrm{fd}} \%<2 \%\right)$.

\subsection{Relaciones entre parámetros magnéticos de los suelos}

Los gráficos entre parámetros magnéticos en diagramas de dispersión son de gran utilidad, ya que permiten comparar las características de las propiedades magnéticas de todos los perfiles en su conjunto (Figura 10). La relación $\chi v s$. MRIS $_{1 \mathrm{~T}}$ es útil para determinar la concentración de minerales ferrimagnéticos y evaluar la presencia de minerales paramagnéticos o antiferrimagnéticos y las mezclas de tamaños (Thompson y Oldfield, 1986). En este diagrama, las
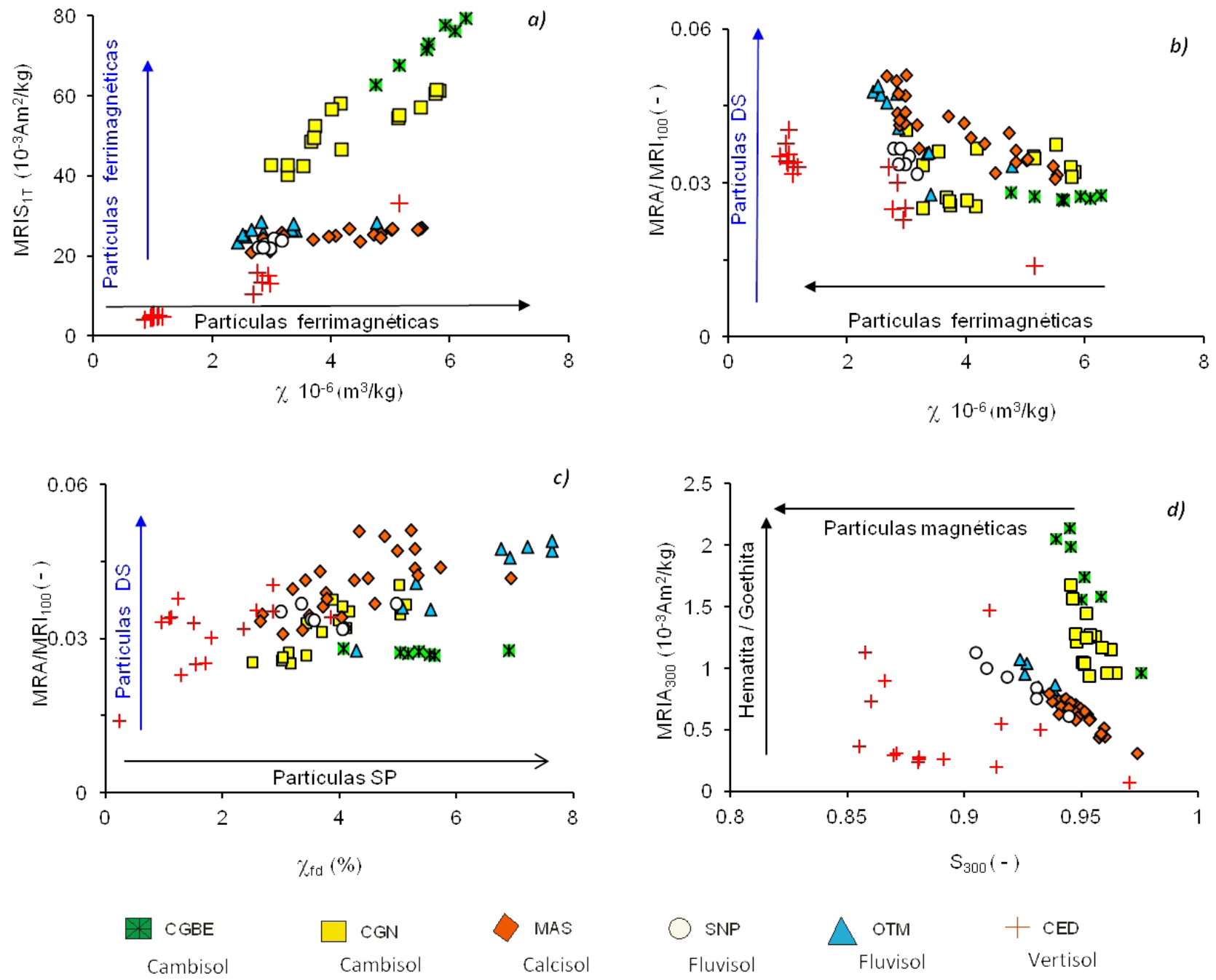

Figura 10. Diagramas de dispersión de algunos parámetros magnéticos. a) $\chi v s . \mathrm{MRIS}_{1 \mathrm{~T}}$ muestra principalmente la concentración de minerales ferrimagnéticos; las concentraciones crecientes de (Ti-)magnetita se ubican hacia el extremo superior derecho; los minerales paramagnéticos contribuyen a la $\chi$ pero no a MRIS $_{1 \mathrm{~T}}$; los minerales de alta coercitividad (hematita y goethita) tienden a ubicarse en la parte baja y derecha del diagrama. b) $\chi v s$. MRA/MRI 100 ilustra la relación entre la concentración magnética y el tamaño de partícula DS-PDS. c) $\chi_{\mathrm{fd}} \% v s$. MRA/MRI ${ }_{100}$ permite analizar las variaciones en las fracciones de partículas más finas, SP y DS, respectivamente d) $\mathrm{S}_{300} v s$. MRIA $_{300}$ muestra la relación entre la concentración absoluta y relativa de minerales de alta coercitividad (hematita y goethita). 
concentraciones crecientes de (Ti-)magnetita se ubican hacia el extremo superior derecho. Los minerales paramagnéticos contribuyen de manera moderada a la $\chi$ pero no a MRIS $_{1 \mathrm{~T}}$, mientras que los minerales de alta coercitividad (hematita/ goethita) tienden a ubicarse en la parte baja y derecha del diagrama. Las magnetitas DS tienden a describir la diagonal de mayor pendiente y las mezclas con altas concentraciones de partículas MD y SP tienden a situarse por debajo de ésta, debido a que contribuyen a la $\chi$ pero en menor medida a MRIS $_{1 \mathrm{~T}}$ (Figura 10a).

En esta figura, los suelos analizados se distribuyen a lo largo de una diagonal en la que el perfil CGBE presenta la mayor concentración de minerales ferrimagnéticos de todos los suelos analizados. Por el contrario, las muestras del perfil CED, que se distribuyen en dos grupos, presentan las menores concentraciones. SNP y OTM se ubican entre los anteriores. El perfil MAS y un grupo de CED se sitúan por debajo de la diagonal, lo que se puede interpretar de forma diferente. Los bajos valores en ambos parámetros en CED sugieren poca presencia de ferrimagnéticos y probablemente mayor contribución de minerales para- y antiferrimagnéticos, mientras que el comportamiento de las muestras de MAS (bajo MRIS $_{1 \mathrm{~T}}$ y alto $\chi$ ) indican una mayor abundancia de minerales paramagnéticos.

La relación entre la concentración magnética y el tamaño de partícula DS se ilustra en el diagrama $\chi$ vs. MRA/MRI ${ }_{100}$ (Figura 10b). En general, los suelos presentan una relación inversa, en donde los suelos con mayor concentración (CGBE, CGN) presentan también la menor abundancia de partículas finas DS, en tanto que el suelo con menor concentración (OTM) presenta una mayor abundancia de DS (MRA/MRI $\left.{ }_{100}>0.05\right)$. Esta relación inversa sugiere que un posible enriquecimiento en la concentración no está asociado a un aumento en partículas finas. Los suelos MAS y CGN presentan la mayor dispersión, entre los valores extremos, lo que indica la mayor variabilidad en el tamaño de partículas en ellos. CED presenta valores bajos e intermedios en MRA/MRI ${ }_{100}$, lo que sugiere variaciones menores en el contenido de DS.

El diagrama $\chi_{\mathrm{fd}} \% v s . \mathrm{MRA} / \mathrm{MRI}_{100}$ permite analizar las variaciones en las fracciones de partículas más finas, SP y DS, respectivamente (Figura 10c). Aunque con cierta dispersión, se observa una relación directa entre ambos parámetros, lo que sugiere que aquellos suelos con la mayor abundancia de partículas DS también presentan la mayor abundancia de partículas ultrafinas SP (OTM). En este diagrama, las muestras de CGN se distribuyen mayormente a lo largo de la diagonal; sin embargo, aunque CGBE y un grupo de MAS contienen de manera moderada partículas $\mathrm{SP}$, tienen una abundancia baja y alta, respectivamente, de partículas DS.

Por otro lado, la relación entre la concentración absoluta y relativa de minerales de alta coercitividad (hematita/ goethita) se muestra en el diagrama $\mathrm{S}_{300}$ vs. MRIA ${ }_{300}$ (Figura 10d). En general, se presenta una relación inversa entre ellos, en la que las muestras de suelos analizados describen dos trayectorias. MAS, OTM y SNP se agrupan en una diagonal de menor pendiente, con valores relativamente bajos de MRIA $_{300}$ y altos de $\mathrm{S}_{300}$, lo que indica bajas concentraciones de hematita/goethita. Por arriba de ellos, a lo largo de una diagonal de mayor pendiente se agrupan CGBE y CGN; sin embargo, éstos presentan altos valores en $\mathrm{S}_{300}$ y en MRIA M00 . Ya que en estos suelos se presenta la mayor concentración absoluta tanto de hematita/goethita como de ferrimagnéticos, es posible que la importancia de los minerales de alta coercitividad decrezca debido a un aumento aún mayor en los ferrimagnéticos de baja coercitividad. Por otra parte, en CED las bajas concentraciones de minerales ferrimagnéticos permiten que las moderadas concentraciones de antiferrimagnéticos resalten en $\mathrm{S}_{300}$.

\subsection{Grado de pedogénesis y su relación con los parámetros magnéticos}

Es evidente que existe una marcada diferenciación de las propiedades magnéticas en función del grado de pedogénesis. Los parámetros magnéticos presentan una estrecha asociación entre las condiciones bajo las que se desarrolla cada suelo, su grado de evolución y los procesos pedogenéticos que transforman la composición y distribución de los minerales magnéticos.

De esta forma, los suelos presentan un comportamiento magnético que varía entre dos extremos, según se aprecia en los diagramas de dispersión (Figura 10): 1) perfiles con mayor concentración y mayor tamaño de partícula (Cambisoles CGBE y CGN); y 2) perfiles con menor concentración y en general menor tamaño de partícula (Fluvisoles SNP y OTM). Entre estos dos extremos se encuentra el Calcisol MAS, en el cual, a medida que se incrementa la concentración, disminuye el contenido de partículas finas. El Vertisol CED, dada su mayor dispersión, no entra en ninguno de los grupos anteriores, pero se observa que tiene una baja concentración y tamaños de partícula magnética MD.

Los suelos con desarrollo moderado (CGBE, CGN), con una edad estimada de 6000 años, muestran que a pesar de tener la misma etapa evolutiva (ambos Cambisoles), presentan propiedades magnéticas ligeramente diferentes. CGBE tiene la mayor concentración tanto de Ti-magnetitas/ Ti-maghemitas como de hematita/goethita (Figura 3) y una mezcla bimodal de tamaños de partícula en la que dominan los granos gruesos MD con cantidades moderadas de SP. Las concentraciones más altas y con partículas más finas se presentan en el horizonte Bw. La disminución de la concentración de minerales ferrimagnéticos y de los granos de tamaño SP en la parte superficial (Figuras 4a, 4b y 4c) puede estar relacionada a un rápido lavado del material con una incipiente eluviación-iluviación, que son controlados por las condiciones de humedad, la estabilidad del sitio (ubicado en un ambiente protegido por vegetación forestal), el buen drenaje, la textura y el alto contenido de la fracción 
limo. Esto permite el lavado hacia la parte baja del suelo, en donde se presenta la zona de mayor concentración y alteración de minerales, formándose óxidos de hierro de tamaño ultrafino (SP), en donde coexisten los minerales magnéticamente suaves y duros. Como consecuencia de este lavado, la parte superficial del perfil se empobrece de partículas SP que resulta en un aumento neto en el tamaño de partícula magnética (Figura 4d).

En CGN la mineralogía magnética es la misma que en CGBE, aunque en menores concentraciones y con mezclas de tamaños MD y proporciones menores de partículas SP (Figuras 5d, 5e y 10c). En contraste con CGBE, en CGN la mayor concentración magnética así como un ligero engrosamiento en el tamaño de partícula se presenta en los horizontes Ap y $\mathrm{Ah}$, en tanto que en el horizonte $\mathrm{AB}$ se observa la disminución en la concentración y un ligero incremento en partículas finas y ultrafinas (Figura 5d y 5e). Aunque los valores de Feo y Fed son similares en el perfil, el cociente Feo/Fed sugiere que es el horizonte Ap el que muestra un bajo intemperismo (Tabla 2), de tal manera que los minerales litogénicos permanecen inalterados. Las observaciones de Cabadas-Báez (2004) apoyan esta interpretación, ya que en Ap se registra un aumento en el contenido de ferromagnesianos, en relación con el resto del perfil.

A diferencia de lo que sucede en las secuencias de loess-paleosuelos que registran un aumento en $\chi$ en la parte superficial, originado por un incremento de minerales ferrimagnéticos y antiferrimagnéticos de tamaño SP a DS, en CGN el realce observado se debe a un aumento en el contenido de partículas gruesas MD, por lo que en este caso no es resultado de la neoformación de minerales magnéticos. La explicación a este comportamiento se asocia a procesos de incorporación de nuevos materiales volcánicos en la superficie (posiblemente ceniza que cayó principalmente en esta superficie y que se mezcló en el horizonte Ap), los cuales contribuyen fuertemente a incrementar la señal. El alto contenido de arena en Ap (50\%) marca claramente esta discontinuidad.

Las diferencias en las propiedades magnéticas de los Cambisoles de Teotihuacán se asocian a: 1) el aporte de materiales alóctonos en la superficie del perfil CGN; y 2) la modificación de la cubierta vegetal, de bosque de encino en CGBE a cultivos de Opuntia en CGN. Este cambio de uso de suelo repercute en el contenido y tipo de materia orgánica, así como en los procesos relacionados con su humificación y mineralización. Este cambio se aprecia claramente en los contenidos de carbono orgánico, los cuales son del $5.5 \%$ para el horizonte Ah en CGBE y de $1.6 \%$ para CGN (Tabla $2)$. Además, el valor del isótopo estable de carbono en cerro Gordo documenta una firma de $\delta^{13} \mathrm{C}$ de $-20 \%$, la cual es diferente para un suelo bajo vegetación forestal (Lounejeva Baturina et al., 2007a, 2007b). La deforestación que ha sufrido la zona de Teotihuacán ha sido documentada desde la época prehispánica, cuando se talaron hasta 70000 ton/año de bosque para la construcción y mantenimiento de la zona ceremonial (Barba-Pingarron, 1995). Es de suponer que las modificaciones en la cubierta vegetal promueven cambios en la humedad del suelo que repercuten en los procesos pedogenéticos, cambiando la mineralogía magnética. En CGBE, esta humedad permite la alteración mineral de los ferromagnesianos, con la consecuente neoformación de óxidos de hierro. Aunque CGN tiene el mismo grado de evolución que CGBE, los cambios de vegetación y estabilidad (con aporte de materiales en esta posición de la ladera) influyen en el desarrollo del suelo y la distribución de los minerales magnéticos.

En MAS, los minerales magnéticos están dominados por Ti-magnetita/Ti-maghemita (y otros minerales paramagnéticos) con una distribución de tamaños de partícula heterogénea (MD-DS-SP) y un contenido ligeramente mayor de partículas DS dentro del horizonte Ap (Figura 6e). La relación Feo/Fed indica que Ap es el horizonte con la menor alteración pedogenética del perfil (Tabla 2). Cabadas-Báez (2004) señala que en este horizonte se presenta una incorporación de material alóctono, heterogéneo, el cual incluye suelos erosionados y redepositados. De acuerdo a sus parámetros magnéticos (Figura 10), MAS se ubica en una zona intermedia entre los perfiles de menor desarrollo (Fluvisoles) y los de moderado desarrollo (Cambisoles). Esto pareciera contradictorio con el grado desarrollo que tienen los suelos, ya que MAS es aparentemente más antiguo que los Cambisoles, dada la edad del material subyacente, fechado en 13358 años cal AP (McClung de Tapia et al., 2005). Sin embargo, esta edad parece ser mucho más antigua que la del suelo. Al respecto, Pustavoitov (2010, comunicación personal) fechó los carbonatos contenidos en Bk y obtuvo una edad de 3900 años. La precipitación de los carbonatos ocurre en fases posteriores a la formación del horizonte $\mathrm{B}$, por lo que se asume que la formación del suelo inicia antes. De acuerdo a los fechamientos de Sedov et al. (2009), se considera que la edad de un Fluvisol calcárico que conforma la cubierta edáfica en Tepexpan (a $15 \mathrm{~km}$ de la zona de estudio) es de 5395 años. Dada esta estimación, se puede suponer que la edad del Calcisol de Maseca es similar a la de los Cambisoles (6500 años).

Las diferencias en las propiedades magnéticas entre los Cambisoles y el Calcisol se deben a las condiciones ambientales marcadamente diferentes en cerro Gordo y Maseca, ya que en las mayores elevaciones son más húmedas y ligeramente más frías (Tabla 1). De hecho, los Calcisoles representan fases evolutivas diferentes a la de los Cambisoles, los cuales requieren de mayor humedad para la alteración de minerales primarios, en tanto que los Calcisoles se forman en regiones con mayor evapotranspiración. Aunque no se tienen datos concretos por la falta de las estaciones meteorológicas, la evapotranspiración en el fondo del valle es 1.3 veces más que la precipitación. A diferencia, en el cerro Gordo, Castilla y Tejero (1983) documentan mayor humedad causada por la presencia de mayor vegetación y el efecto de sombra que se tiene. 
Los perfiles más jóvenes y de menor desarrollo (SNP y OTM) presentan características magnéticas similares. Ambos poseen bajas concentraciones de minerales magnéticos en los que dominan Ti-magnetita/Ti-maghemita con cantidades menores de hematita/goethita, que son aún menores en OTM (Figuras 10a y 10d), y mezclas de tamaños MD-DS-SP. La mayor diferencia entre ellos es el realce en $\chi$ en la superficie de OTM que está ausente en SNP, a pesar de ser suelos con pedogénesis similar, y en la distribución del contenido en partículas SP (Figura 10c). En SNP hay un aumento moderado de partículas SP en la parte superior (Figuras 7d), en tanto que en OTM el horizonte $\mathrm{AB}$ presenta un contenido alto de partículas SP, y muy alto en la parte superficial del horizonte Ap (Figura 8d).

La diferencia en el realce en $\chi$ entre los Fluvisoles OTM y SNP puede deberse al tipo de material que se aporta a cada suelo, ya que ambos son formados por procesos de aluvionamiento, es decir por incorporación de materiales aluviales, los cuales pueden transportar partículas de fuentes heterogéneas. Cabadas-Báez (2004), McClung de Tapia et al. (2003 y 2005), Solleiro-Rebolledo et al. (2006) y RiveraUria et al. (2007) reportan que estos perfiles muestran rasgos contrastantes. Por un lado, tienen minerales volcánicos poco alterados que revelan su bajo grado de desarrollo, con minerales caoliníticos de mayor intemperismo. Esto, aunado a la inversión en los fechamientos en SNP (Rivera-Uria et al., 2007), permite suponer que hay aporte de restos de suelos, erosionados de las partes más altas. Cabadas-Báez (2004) y Solleiro-Rebolledo et al. (2006) documentan la presencia de paleosuelos formados en ambientes de mayor humedad (Luvisoles) en CGN y MAS, cuya señal magnética se ha mezclado con la observada en SNP. Este comportamiento diferencial de los suelos puede ayudar a las interpretaciones paleoambientales, ya que la influencia de la cantidad de humedad en los perfiles es importante para definir la alteración de los minerales volcánicos y por ende de la formación de hierro en el suelo y la dinámica de su mineralogía magnética.

La característica más notoria de CED es la menor concentración de minerales ferrimagnéticos de todos los perfiles analizados. Así mismo, este suelo presenta en el horizonte Ap un incremento tanto de minerales ferrimagnéticos como de antiferrimagnéticos. Al contrario de lo que sucede en CGBE, donde la presencia relativamente alta de minerales de alta coercitividad (hematita/goethita) queda enmascarada por la abundancia aún mayor de minerales ferrimagnéticos, en CED las bajas concentraciones de ferrimagnéticos permiten que los minerales antiferrimagnéticos resalten en S300. Como ya se ha mencionado, CED es un suelo cuyo comportamiento magnético es diferente al registrado en el resto de los suelos estudiados en el valle de Teotihuacán. Sin embargo, en un estudio preliminar, Solleiro-Rebolledo et al. (2011) señalan la presencia de un incremento notorio en $\chi$ en la parte inferior del perfil SNP, precisamente en la zona en la que se encuentra un paleosuelo de color oscuro con fuertes rasgos vérticos. Este paleosuelo es análogo al perfil CED, el cual aparece en superficie a consecuencia de procesos de erosión que han eliminado los materiales superficiales, dejando sólo restos de un horizonte Ap. Es decir, $\mathrm{Ah}_{1} \mathrm{y} \mathrm{Ah}_{2}$ son horizontes de un paleovertisol formado hace aproximadamente 3000 años que ahora se encuentra casi en superficie, sólo sepultado por el horizonte Ap. Este horizonte Ap puede ser correlacionado con los Fluvisoles SNP y OTM. A diferencia de estos últimos, el paleosuelo muestra un mayor grado de desarrollo. Las propiedades vérticas (fracturamiento debido a los procesos de expansión y contracción estacionales) promueven la mezcla de materiales entre los horizontes, por lo que es de suponer que la heterogeneidad mostrada en los parámetros magnéticos se deba a estos procesos de argiloturbación continua.

\section{Conclusiones}

No obstante que las condiciones del clima regional y el tipo de material parental (materiales volcánicos andesíticobasálticos) son similares, los suelos presentan diversos estados de evolución como consecuencia de diferencias en otros factores de formación: relieve, edad y vegetación, así como cambios en el uso del suelo. Estas diferencias se ven reflejadas en sus propiedades magnéticas, a partir de las cuales es posible agrupar a los suelos de acuerdo con su grado de evolución y de las condiciones ambientales. 1) Suelos en etapas de evolución moderada: Cambisoles (CGBN y CGN) con mayor concentración magnética y mayor tamaño de partícula; y 2) suelos en etapas incipientes de evolución: Fluvisoles SNP y OTM, con bajas concentraciones y tamaño de partículas magnéticas más finas. Entre estos dos extremos se encuentra el suelo de mayor evolución, Calcisol MAS, en el que existe una correlación inversa entre la concentración y el contenido de partículas finas. Esto se debe a que su pedogénesis demuestra procesos relacionados a cambios estacionales marcados en el grado de humedad, lo que conlleva a la precipitación de carbonatos en el suelo, proceso ausente en los Cambisoles, ya que éstos están ubicados en posiciones de mayor altitud y humedad con buen drenaje.

Los suelos con desarrollo moderado (CGBE, CGN), con una edad estimada de 6500 años y a pesar de tener la misma etapa evolutiva, presentan diferencias en sus propiedades magnéticas. En CGBE, la densa cubierta vegetal, el mayor drenaje y rápido lixiviado en la parte superior favorecen el intemperismo de los minerales volcánicos primarios y la neoformación y acumulación de óxidos de hierro, magnetitas y hematitas/goethitas, en el horizonte Bw. La disminución de la concentración y de granos SP en la parte superficial puede relacionarse a la disolución e hidrólisis durante la descomposición de la materia orgánica, que actúa preferencialmente en granos finos, lo que resulta en un aumento neto en el tamaño de partícula magnética.

A diferencia de lo que sucede en las secuencias de 
loess-paleosuelos que registran un aumento en $\chi$ en la parte superficial originado por la neoformación de partículas SP a DS, en CGN el realce en la concentración coincide con un aumento en el tamaño de partícula. La deforestación y la cultivación en la ladera donde se encuentra CGN ocasionan cambios en los regímenes de humedad del suelo y promueven la erosión de sus partículas y el redepósito de materiales erosionados de la parte superior. Por lo tanto, se considera que en CGN el incremento en la concentración de minerales magnéticos se debe a la incorporación de material alóctono grueso y no a la neoformación de finos. Las condiciones ambientales marcadamente diferentes en cerro Gordo y Maseca controlan las diferencias en las propiedades magnéticas entre los contemporáneos Cambisoles y el Calcisol, ya que los Cambisoles requieren de mayor humedad para la alteración de minerales primarios, en tanto que los Calcisoles se forman en regiones con mayor evapotranspiración.

Los perfiles más jóvenes y de menor desarrollo, los Fluvisoles SNP y OTM, poseen bajas concentraciones de minerales magnéticos en donde dominan las partículas gruesas. Una diferencia importante observada entre OTM y SNP es la ausencia del realce en $\chi$ en SNP, a pesar de ser suelos con pedogénesis similar. La diferencia puede deberse al tipo de material que se aporta a cada suelo, ya que ambos son formados por la incorporación de materiales aluviales con partículas de características heterogéneas. El perfil CED representa un caso particular de los suelos del valle de Teotihuacán, cuyas propiedades magnéticas difieren considerablemente de las encontradas en el resto. Dado que se trata de un paleosuelo formado hace aproximadamente 3000 años atrás (Solleiro-Rebolledo et al., 2011), dichas propiedades son producto de condiciones ambientales diferentes a las que se presentan hoy en día en la región. $\mathrm{Si}$ bien existe un realce en la concentración de minerales magnéticos en el horizonte superficial, la ausencia de minerales de tamaño SP así como sus propiedades vérticas, indican que el agrietamiento y la mezcla de materiales -y no la pedogénesis- son responsables de este comportamiento

En síntesis, las propiedades magnéticas de los suelos de Teotihuacán varían de acuerdo a su grado de evolución y gradiente de humedad, semejante a lo reportado en una climosecuencia de las pampas argentinas (Bartel et al., 2011). Los análisis aquí presentados muestran el potencial de los estudios de propiedades magnéticas de suelos, utilizando no sólo la $\chi$, sino la combinación de varios parámetros de remanencias magnéticas y otros indicadores pedogenéticos, en la reconstrucción de las condiciones ambientales del pasado.

\section{Agradecimientos}

Los resultados que se presentan en este documento forman parte del trabajo de investigación doctoral de $\mathrm{J}$. Rivas, quien gozó de una beca otorgada por CONACYT. Esta investigación es parte de los proyectos PAPIIT IN112205 y CONACYT 43746-1. Se agradece la colaboración de H. Cabadas, C. Jasso, J. Gama, Y. Rivera, A. González, G. Vázquez, G. Rivas, H. Reyes, J. Barrientos, C. Borunda ${ }^{\dagger}$ y J.A. González, por el apoyo brindado en el trabajo de campo, laboratorio, interpretación de los datos y elaboración de figuras. Los autores agradecen a la Dra. A.A. Bartel y el Dr. M.A.E. Chaparro por sus valiosas observaciones y sugerencias que contribuyeron a mejorar este documento

\section{Referencias}

Aragón, R., Buttrey, D.J., Shepherd, J.P., Honig, J.M., 1985, Influence of nonstoichiometry on the Verwey transition: Physical Review B, 31, 430-436.

Barba-Pingarron, L.A., 1995, El impacto humano en la paleogeografía de Teotihuacán: Cd. de México, Faculta de Filosofía y Letras, Universidad Nacional Autónoma de México, tesis de doctorado, 79 p.

Bartel, A.A., Bidegain, J.C., Sinito, A.M., 2011, Magnetic parameter analysis of a climosequence of soils in the southern Pampean Region, Argentina: Geofísica Internacional, 50, 9-22.

Bidegain, J.C., Rico, Y., Bartel A., Chaparro, M.A.E., Jurado, S., 2009, Magnetic parameters reflecting pedogenesis in Pleistocene loess deposits of Argentina: Quaternary International, 209, 175-186.

Cabadas-Báez, H.V, 2004, Paleosuelos como indicadores de cambio ambiental en el Cuaternario superior: El caso del valle de Teotihuacán: Cd. de México, Facultad de Ingeniería, Universidad Nacional Autónoma de México, tesis de licenciatura, 167 p.

Cabadas-Báez, H.V., 2007, Paleosuelos del centro de México como indicadores de cambios ambientales ocurridos durante los últimos 30000 años: Cd. de México, Instituto de Geología, Universidad Nacional Autónoma de México, tesis de maestría, 168 p.

Castilla, M.E., Tejero, J.D., 1983, Estudio florístico del cerro Gordo (próximo a San Juan Teotihuacán) y regiones aledañas: Cd. de México, Escuela Nacional de Estudios Profesionales Iztacala, Universidad Nacional Autónoma de México, tesis de licenciatura, $128 \mathrm{p}$.

Chaparro, M.A.E, 2006, Estudio de parámetros magnéticos de distintos ambientes relativamente contaminados en Argentina y Antártida: Cd. de México, Instituto de Geofísica, UNAM, 107 p.

Collinson, D. W., 1983, Methods in Rock Magnetism and Palaeomagnetism: Techniques and Instrumentation: Londres, Chapman and Hall, 503 p.

Dearing, J.A., 1994, Environmental Magnetic Susceptibility: Using the Bartington MS2 System: Lincoln, Reino Unido, Chi Publishing, $111 \mathrm{p}$.

Dearing, J.A., Bird, P.M., Dann R.J.L., Benjamin S.F., 1997, Secondary ferrimagnetic minerals in Welsh soils: a comparison of mineral magnetic detection methods and implications for mineral formation: Geophysical Journal International, 130, 727-736.

Dunlop, D., Özdemir, Ö., 1997, Rock Magnetism: Fundamentals and Frontiers: Nueva York, Cambridge University Press, 573 p.

Gama-Castro, J., McClung de Tapia, E., Solleiro-Rebolledo, E., Ibarra, E., Sedov, S., Jasso-Castañeda, C., Vallejo-Gómez, E., Pi-Puig, T., Cabadas-Báez, H., 2005a, Incorporation of ethnopedological knowledge in the study of soils in the Teotihuacan valley, Mexico: Eurasian Soil Science, 38, 95-98.

Gama-Castro, J.E., Solleiro-Rebolledo, E., McClung E., Villalpando, J.L., Sedov, S., Jasso-Castañeda, C., Palacios-Magorya, S., Hernández, D., 2005b, Contribuciones de la ciencia del suelo a la investigación arqueológica: El caso de Teotihuacán: Terra Latinoamericana, 23, 1-11.

García, E., 1968, Clima Actual de Teotihuacán: Materiales para Arqueología de Teotihuacán: INAH, México, 9-28.

García, E., 1988, Modificaciones al Sistema de Clasificación Climática de Köppen: Universidad Nacional Autónoma de México, México, Instituto de Geografía, México, pp. 219. 
García-Palomo, A., Macías, J.L., Tolson, G., Valdez G., Mora J.C., 2002, Volcanic stratigraphy and geological evolution of the Apan region, east-central sector of the Trans-Mexican Volcanic Belt: Geofísica Internacional, 41, 133-150.

Geiss, C.E., Zanner C.W., 2006, How abundant is pedogenic magnetite? Abundance and grain size estimates for loessic soils based on rock magnetic analyses: Journal of Geophysical Research, 111, doi:10.1029/2006JB004564.

Geiss, C.E., Zanner, C.W., 2007, Sediment magnetic signature of climate in modern loessic soils from the Great Plains: Quaternary International, $162 / 163,97-110$

Geiss, C.E., Zanner, W., Banerjee, S.K., Joanna, M., 2004, Signature of magnetic enhancement in a loessic soil in Nebraska, United States of America: Earth and Planetary Science Letters, 228, 355-367.

Geiss, C.E., Egli, R. Zanner, C.W., 2008, Direct Estimates of Pedogenic Magnetite as a Tool to Reconstruct Past Climates From Buried Soils: Journal of Geophisycal Research, 113, doi:10.1029/2008JB005669.

Grimley, D.A., Vepraskas, M.J., 2000, Magnetic susceptibility for use in delineating hydric soils: Soil Science Society of America Journal, 64, 2174-2180.

Grimley, D.A., Arruda, N.K., Bramstedt, M.W., 2004, Using magnetic susceptibility to facilitate more rapid, reproducible and precise delineation of hydric soils in the midwestern USA: Catena, 58, 183-213.

Han, J., Lu, H., Wu, N., Guo, Z., 1996, The Magnetic Susceptibility of Modern soils in China and its Use for Paleoclimate Reconstructions: Studia Geophysica et Geodetica 40, 262-275.

Heller, F., Evans, M. E., 1995, Loess magnetism: Review of Geophysics, 33, 211-240.

Hunt, C.P., Banerjee, S.K., Han, J., Solheid, P.A., Oaches, E., Sun, W., Liu, T., 1995, Rock-magnetic proxies of climate change in the loess-paleosol sequences of the western Loess Plateau of China: Geophysical Journal International, 123, 232-244.

Instituto Nacional de Estadística, Geografía e Informática (INEGI), 1999, Carta Edafológica Texcoco E14B21, 1:50000: Ciudad de México, Instituto Nacional de Estadística, Geografía e Informática, 1 mapa.

Jordanova, N., Kovacheva, M., Hedley, I., Kostadinova, M., 2003, On the suitability of baked clay for archaeomagnetic studies as deduced from detailed rock-magnetic studies: Geophysical Journal International, 153, 146-158.

Jordanova, N., Kovacheva, M., Kostadinova, M., 2004, Archaeomagnetic investigation and dating of Neolithic archaeological site (Kovachevo) from Bulgaria: Physics of the Earth and Planetary Interiors, 147, 89102.

Liu, Q., Deng, Ch., Torrent, J., Zhu, R., 2007, Review of recent developments in mineral magnetism of the Chinese loess: Quaternary Science Review, 26, 368-385.

Liu, Q., Hu, P., Torrent, J., Barrón, V., Zhao, X., Jiang, Z, Su, Y., 2010, Environmental magnetic study of a Xeralf chronosequence in northwestern Spain: Indicators for Pedogenesis: Palaeogeography, Palaeoclimatology, Palaeoecology, 293, 144-156.

Lounejeva Baturina, E., Morales Puente, P., Cabadas Báez, H.V., Cienfuegos Alvarado, E., Sedov, S., Vallejo Gómez, E., Solleiro Rebolledo, E., 2007a, Late Pleistocene to Holocene environmental changes from $\delta^{13} \mathrm{C}$ determinations in soils at Teotihuacan, Mexico: Geofísica Internacional, 45, 85-98.

Lounejeva Baturina, E., Morales Puente, P., Cienfuegos Alvarado, E., Sedov, S., Solleiro Rebolledo, E., 2007b, Late Quaternary environment in the Teotihuacan Valley, Mexico, inferred from $\delta^{13} \mathrm{C}$ in soils: Society for Archaeological Sciences Bulletin, 30, 6-11.

Lu, S.G., Xue, Q.F., Zhu, L., Yu, J.Y., 2008, Mineral magnetic properties of a weathering sequence of soils derived from basalt in Eastern China: Catena, 73, 23-33.

Lugo Hubp, J., 1984, Geomorfología del Sur de la Cuenca de México: Cd. de México, Instituto de Geografía, Universidad Nacional Autónoma de México, $95 \mathrm{p}$.

Maher, B.A., 1986, Characterisation of soils by mineral magnetic measurements: Physics of the Earth and Planetary Interiors, 42, 76-92.
Maher, B.A., 1998, Magnetic properties of modern soils and Quaternary loessic paleosols: paleoclimatic implications: Paleogeography, Paleoclimatology, Paleoecology, 137, 25-54.

Maher, B.A., Thompson, R., Zhou, L.P., 1994, Spatial and temporal reconstructions of changes in the Asian paleomonsoon: A new mineral magnetic approach: Earth and Planetary Science Letters, $125,461-471$.

Maher, B.A., Alekseev, A., Alekseeva, T., 2002, Variation of soil magnetism across the Russian steppe: its significance for use of soil magnetism as a palaeorainfall proxy: Quaternary Science Reviews, 21, 15711576.

McClung de Tapia, E., Solleiro-Rebolledo, E., Gama-Castro, J., Villalpando, J.L., Sedov, S, 2003, Paleosols in the Teotihuacan valley, Mexico: Evidence for paleoenvironment and human impact: Revista Mexicana de Ciencias Geológicas 20, 270-282.

McClung de Tapia, E., Dominguez-Rubio, I., Gama-Castro, J., SolleiroRebolledo, E., Sedov, S., 2005, Radiocarbon dates from soil profiles in the Teotihuacan valley, Mexico: Indicators of Geomorphological Processes: Radiocarbon, 47, 159-175.

Miyahara, Y., 1972, Impurity effects on the transition temperature of magnetite: Journal of the Physical Society of Japan, 32, 629-634.

Morin, F.J., Moskowitz, B.M., Franke, R.B.1., 1950, Magnetic susceptibility of $\mathrm{aFe}_{2} \mathrm{O}_{3}$ and $\mathrm{aFe}_{2} \mathrm{O}_{3}$ with added titanium: Physical Review, 78, $819-820$.

Mullins, C.E., 1977, Magnetic susceptibility of the soil and its significance in soil science - a review: Journal of Soil Science, 28, 223-246.

Munsell Soil Charts, 2000, Macbeth Division of Kollmorgen.

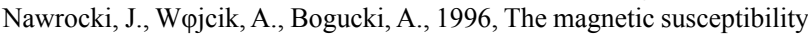
record in the Polish and western Ukrainian loess-paleosol sequences conditioned by paleoclimate: Boreas 25, 161-169.

Oaches, E.A., Banerjee, S.K., 1996, Rock-magnetic proxies of climate change from loess-paleosol sediments of the Czech Republic: Studia Geophysica et Geodaetica, 40, 287-300.

Opdyke, N.D., Channell, J.E.T., 1996, Magnetic Stratigraphy: San Diego, California, Academic Press, 346 p.

Orgeira, M.J., Walther, A.M., Vásquez, C.A., Di Tommaso, I., Alonso, S., Sherwood, G., Yuguan, H., Vilas, J.F.A., 1998, Mineral magnetic record of paleoclimate variation in loess and paleosol from the Buenos Aires formation (Buenos Aires, Argentina): Journal of South American Earth Sciences, 11, 561-570.

Orgeira, M.J., Walther, A.M., Tofalo, R.O., Vásquez, C., Berquo, T., Favier Dobois, C., Bonhel, H., 2003, Environmental magnetism in fluvial and loessic Holocene sediments and paleosols from the Chacopampean plain (Argentina): Journal of South American Earth Sciences, 16, 259-274.

Orgeira, M.J., Pereyra, F.X., Vásquez, C., Castañeda, E, Compagnucci, R., 2008, Rock magnetism in modern soils, Buenos Aires Province, Argentina: Journal of South American Earth Sciences, 26, 217-224.

Ortega-Guerrero, B., Sedov, S., Solleiro-Rebolledo, E., Soler, A., 2004, Magnetic mineralogy in Barranca Tlalpan exposure paleosols, Tlaxcala, Mexico: Revista Mexicana de Ciencias Geológicas, 21, 120-132.

Özdemir, Ö., Dunlop, D.J., 1996, Thermoremanence and Néel temperature of goethite: Geophysical Research Letters, 23, 921-924.

Özdemir, Ö., Dunlop, D.J., Moskowitz B.M., 1993, The effect of oxidation on the Verwey transition in magnetite: Geophysical Research Letters, 20, 1671-1674.

Porter, S.C., Hallet, B., Wu, X., An, Z., 2001, Dependence of near surface magnetic susceptibility on dust accumulation rate and precipitation on the Chinese Loess Plateau: Quaternary Research, 55, 271-283.

Rivas-Ortiz, J.F., 2003, Propiedades magnéticas de paleosuelos cuaternarios del Nevado de Toluca y su aplicación en la reconstrucción paleoambiental: Cd. de México, Facultad de Ingeniería, Universidad Nacional Autónoma de México, tesis de licenciatura, 91 p.

Rivas-Ortiz, J.F., 2006, Análisis de magnetismo de rocas en paleosuelos pleistocénicos de origen volcánico y eólico, como una herramienta alterna en la interpretación paleoambiental: Cd. de México, México, Instituto de Geofísica, Universidad Nacional Autónoma de México, tesis de maestría, $154 \mathrm{p}$. 
Rivas-Ortiz, J., Ortega, B., Sedov, S., Solleiro, E., Sycheva, S., 2006, Rock magnetism and pedogenetic processes in Luvisol profiles: Examples From central Russia and central Mexico: Quaternary International, $156 / 157,212-223$.

Rivera-Uria, Y., 2008, Impacto del desarrollo cultural prehispánico en la cubierta edáfica del valle de Teotihuacán: Cd. de México, Instituto de Geología, Universidad Nacional Autónoma de México, tesis de maestría, $124 \mathrm{p}$.

Rivera-Uria, M.Y., Sedov S., Solleiro-Rebolledo, E., Pérez-Pérez, J., McClung de Tapia, E., González, A., González, A., Gama-Castro, J., 2007, Degradación ambiental en el valle Teotihuacan: evidencias geológicas y paleopedológicas: Boletín de la Sociedad Geológica Mexicana, 59, 203-217.

Schellenberger, A., Heller, F., Veit, H., 2003, Magnetostratigraphy and magnetic susceptibility of the Las Carreras loess-paleosol sequence in Valle de Tafí, Tucumán, NW-Argentina: Quaternary International, 106/107, 159-167.

Sedov, S., Lozano-García, S., Solleiro-Rebolledo, E., McClung de Tapia, E., Ortega-Guerrero, B., Sosa-Nájera, S., 2009, Tepexpan revisited: A multiple proxy of local environmental changes in relation to human occupation from a paleolake shore section in Central Mexico: Geomorphology, 122, 309-322.

Solleiro-Rebolledo, E., Sedov, S., McClung de Tapia, E., Cabadas-Báez, H., Gama-Castro, J., Vallejo-Gómez, E., 2006, Spatial variability of environment change in the Teotihuacan Valley during the Late Quaternary: Paleopedological inferences: Quaternary International, $156 / 157,13-31$

Solleiro-Rebolledo, E., Sycheva, S., Sedov, S., McClung de Tapia, E., Rivera-Uria, Y., Salcido-Berkovich, C., Kuznetsova, A., 2011, Fluvial processes and paleopedogenesis in the Teotihuacan Valley, México: Responses to late Quaternary environmental changes: Quaternary International, 233, 40-52.
Tarling, D.H., 1983, Palaeomagnetism: Principles and Applications in Geology, Geophysics and Archeology: Londres, Chapman and Hall, 379 p.

Terhorst, B., Appel, E., Werner, A., 2001, Palaeopedology and magnetic susceptibility of a loess-palaeosol sequence in southwest Germany: Quaternary International, 76/77, 231-240.

Thompson, R., Oldfield F., 1986, Environmental Magnetism: Londres, Allen \& Unwin, 227 p.

Tite, M.S., Linington, R.E., 1975, Effect of climate on the magnetic susceptibility of soils: Nature, 256, 565-566.

Vásquez, C., Walther, A.M., Orgeira, M.J, Di Tommaso, I.M., Lippai, H., Alonso, S., Vilas, J.F.A., 1998, Magnetismo de rocas y su aplicación a paleosuelos: estudio piloto en una secuencia loess-paleosuelo de la región chacopampeana: Revista de la Asociación Geológica Argentina, 53, 83-90.

Vázquez-Sánchez, E., Jaimes-Palomera, R., 1989, Geología de la cuenca de México: Geofísica Internacional, 28, 133-189.

Verosub, K.L., Fine, P., Singer M.J., TenPas, J., 1993, Pedogenesis and paleoclimate: Interpretation of the magnetic susceptibility record of the Chinese loess-paleosol sequences: Geology, 21, 1011-1014.

World Reference Base for Soil Resources (WRB), 2006, World Reference Base for Soil Resources 2006 (World Soil Resources Reports No. 103): Roma, Food and Agriculture Organization of the United Nations, 132 p.

Manuscrito recibido: Junio 15, 2011

Manuscrito corregido recibido: Noviembre 11, 2011

Manuscrito aceptado: Diciembre 1, 2011 